\title{
Pressure and spanwise velocity fluctuations in turbulent channel flows: Logarithmic behavior of moments and coherent structures
}

\author{
Ali Mehrez, ${ }^{1, *}$ Jimmy Philip, ${ }^{2}$ Yoshinobu Yamamoto, ${ }^{3}$ and Yoshiyuki Tsuji ${ }^{1}$ \\ ${ }^{1}$ Department of Energy Engineering and Science, Nagoya University, Chikusa-ku, Furo-cho 464-8603, Japan \\ ${ }^{2}$ Department of Mechanical Engineering, The University of Melbourne, Parkville, Victoria 3010, Australia \\ ${ }^{3}$ Department of Mechanical Engineering, University of Yamanashi, Takeda, Kofu, Yamanashi 400-8510, Japan
}

(Received 16 September 2018; published 2 April 2019)

\begin{abstract}
We study the logarithmic behavior of the pressure variance $\left\langle p^{+^{2}}\right\rangle$ from the datasets obtained from direct numerical simulations of turbulent channel flow for friction Reynolds number $\operatorname{Re}_{\tau}$ up to 4000. The higher-order moments of $p$ were found to follow logarithmic behaviors at the same distances from the wall where $\left\langle p^{+^{2}}\right\rangle$ shows its $\log$ profile. The same results have been confirmed for the spanwise velocity fluctuations $w$ at the same Reynolds numbers, with both $p$ and $w$ following a super-Gaussian behavior. The minimum Reynolds number for $\left\langle p^{+^{2}}\right\rangle$ and $\left\langle w^{+^{2}}\right\rangle \log$ profiles to appear is $\operatorname{Re}_{\tau} \approx 500$, where flow structures $\mathcal{O}(h)$ or less were found to significantly contribute to these profiles. The configuration of the hairpin eddy structures obtained from the conditional sampling at different wall-normal locations showed a strong link between $p$ and $w$ fluctuations. Positive pressure fluctuations are located between the legs of the hairpin eddy, while the negative pressure fluctuations are consistent with the head part of the hairpin eddy. Positive and negative spanwise velocity fluctuations are strongly positioned with the legs of the hairpin eddy, consistent with the counter-rotating motion resulting from the eddy legs. The structures were also found to be geometrically self-similar such that their length and their width increase linearly with the distance from the wall.
\end{abstract}

DOI: 10.1103/PhysRevFluids.4.044601

\section{INTRODUCTION}

The original idea of Townsend's attached eddy hypothesis [1] to predict the turbulence intensity in wall turbulence was extended by Perry et al. [2]. Both of them reported that the turbulent eddies have a population density that is inversely proportional to the distance from the wall. This leads to the result that the intensities of turbulent fluctuations of the wall-parallel velocities, streamwise intensity $\left\langle u^{+^{2}}\right\rangle$, and spanwise intensity $\left\langle w^{+^{2}}\right\rangle$ are logarithmically proportional to the distance from the wall. The relations are expressed as

$$
\begin{gathered}
\left\langle u^{+^{2}}\right\rangle=-A_{u} \ln (y / h)+B_{u}, \\
\left\langle w^{+^{2}}\right\rangle=-A_{w} \ln (y / h)+B_{w} .
\end{gathered}
$$

Here, $A_{u}$ and $A_{w}$ are universal constants, while $B_{u}$ and $B_{w}$ are constants that depend on the boundary conditions of the flow field [2,3], and are thus not expected to be universal. Here, $y$ represents the distance from the wall, $h$ is the outer length scale, which in this paper is the turbulent

*mehrez-ali16@ees.nagoya-u.ac.jp 
channel half depth, and the superscript + indicates normalization by the inner variables (with the kinematic viscosity $v$ and the friction velocity $u_{\tau}$ ). A recent review of the attached eddy model was presented by Marusic and Monty [4].

Interestingly, the pressure intensity $\left\langle p^{+^{2}}\right\rangle$ was also found to follow a similar log trend (e.g., Jiménez and Hoyas [5] and Tsuji et al. [6]):

$$
\left\langle p^{+^{2}}\right\rangle=-A_{p} \ln (y / h)+B_{p} .
$$

It is important to note that this distribution is not directly derived from the attached eddy framework of Townsend [1]; rather, it is an empirical observation.

Although there is much interest in the wall pressure scaling, the present paper is related to the pressure distribution inside the flow. Even so, we provide a brief introduction to the wall pressure scaling too. Townsend [1] implied that the pressure intensity at the wall tends to increase logarithmically with Reynolds number once it is scaled with the shear stress. This logarithmic growth of the wall pressure had been emphasized for a long time from both experiments [7-10] and direct numerical simulation (DNS) [5,11,12]. Further, Tsuji et al. [10] reported that the same tendency is not just applied for the wall pressure intensity, but it is also applied for the pressure intensity at its peak position that occurs at $y^{+} \approx 30$.

Later, Jiménez and Hoyas [5] indicated that this logarithmic growth of the pressure at the wall, and generally in the buffer region, is a consequence of the accumulation of attached selfsimilar eddies with sizes $\mathcal{O}(y)$. Their analysis of the two-dimensional (2D) spectra of the pressure fluctuations of boundary layers and channels from DNS datasets indicated a collapse of the spectra in the long-wavelength limits when they are scaled with $h$. Hence, the pressure fluctuations have an attached-wall character like that of the wall-parallel velocity fluctuations. Consequently, it is quite plausible and indeed consistent with the observations that the pressure fluctuations possess a logarithmic intensity profile in the overlap layer with their intensities increasing linearly with $\ln \left(\operatorname{Re}_{\tau}\right)$ close to the wall. Here, $\operatorname{Re}_{\tau}=h u_{\tau} / v$ is the friction Reynolds number. Additionally, from the analysis of Poisson's pressure equation by Green's function, Jiménez and Hoyas [5] identified two mean structures concerned with the pressure fluctuations. The first one is a very strong localized structure in the near-wall and logarithmic regions, while the second one is a large-scale structure $\mathcal{O}(h)$ that extends across the boundary layer. However, Jiménez and Hoyas [5] did not explore these structures and the contributions to the $\log$ trend of $\left\langle p^{+^{2}}\right\rangle$ because their focus was on the spectral analysis of the pressure fluctuations without invoking any specific model. Upon discussing the three-dimensional (3D) two-point correlations for boundary layers and channels, Sillero et al. [13] quantified the first and second structures in terms of strong and weak correlations, respectively.

Recently, Tsuji et al. [6] attempted to interpret the structures behind the log trend of $\left\langle p^{+^{2}}\right\rangle$. They divided the instantaneous pressure field with various levels of streamwise wavelength and observed the pressure intensity profiles for larger and smaller scales of motion than a cutoff wavelength. As they reduced the value of the streamwise cutoff wavelength, the contribution from the smaller scales of motion to the log trend of $\left\langle p^{+{ }^{2}}\right\rangle$ increased. Although they investigated the interaction between large and small scales of motion by means of an amplitude modulation procedure, their pointwise measurements did not enable them to explore the coherent structures associated with the pressure logarithmic intensity profile.

Even though the pressure fluctuations are related to the three velocity components across the boundary layer via Poisson's pressure equation, the logarithmic intensity profiles that are predicted for $u$ and $w$ components cannot be employed to derive a similar relation to $\left\langle p^{+^{2}}\right\rangle$. In this paper, we focus on the logarithmic intensity profile of the pressure variance as an empirically obtained statistical quantity in turbulent channel flows along with the structures responsible for generating it.

In the case of the $u$ component, several experimental studies have revealed that we need very high Reynolds number to observe the logarithmic intensity profile [14-16], and recently Yamamoto and Tsuji [17] from DNS stated that Eq. (1) appears for channels at $\operatorname{Re}_{\tau}=8000$. For the spanwise velocity the situation is different, as various experimental studies [18-22] have confirmed Eq. (2) 
at lower Reynolds number compared to that of $u$ fluctuations, however without discussing the turbulent structures related to this logarithmic intensity profile. DNS based studies [5,12,23-26] state that both spanwise velocity and pressure fluctuations have their own logarithmic intensity profiles given by Eqs. (2) and (3), respectively, within the same range of Reynolds numbers. Jiménez and Hoyas [5] indicate the similarity of the 2D spectra for $p$ and $w$. Both spectra scale with $h$ in the long-wavelength limits, and they are aligned along the equilateral line $\lambda_{x} \approx \lambda_{z} \approx 3 y$, where $\lambda_{x}$ and $\lambda_{z}$ are the streamwise and spanwise wavelengths, respectively. The spectra of the wall-normal velocity component $v$ are also found to be aligned along the same equilateral line [5]. However, $v$ spectra collapse with $y$ and lack the scaling with $h[2,5]$. Hence, this component does not produce a $\log$ profile for its variance [5]. Here, we study the pressure intensity log profile by discussing the statistical similarity between the pressure and spanwise velocity fluctuations.

There are several issues that motivated us to conduct this study. First, despite the detailed analysis of the pressure and spanwise velocity fluctuations by Jiménez and Hoyas [5] from the spectral point of view as well as from the 3D two-point correlation reported by Sillero et al. [13], none of these studies examined the structures related to $p$ or $w$ intensity log profiles. Sillero et al. [13] and recently Jiménez [27] suggest definite structures related to the spanwise velocity component by combining the two-point correlations of $v$ and $w$ to be quasistreamwise rollers closely associated with the logarithmic region and scaled in outer units. However, these studies do not indicate whether these structures are related to the logarithmic intensity profile of the spanwise velocity fluctuations. Hence, in this paper, the structures associated with $p$ and $w$ intensity log profiles are explored along with an investigation of the Reynolds number dependency.

Second, none of the aforementioned studies [5,12,23-26] discusses the Reynolds number dependence of the bounds of the logarithmic regions of the pressure and spanwise velocity fluctuations in turbulent channel flow. Yamamoto and Tsuji [17] recently reported that the logarithmic region of $\left\langle u^{+^{2}}\right\rangle$ does not overlap that of the mean velocity $U^{+}$at high Reynolds number $\operatorname{Re}_{\tau}=8000$ of turbulent channel flow. So, it is not clear whether the pressure and spanwise velocity intensity $\log$ regions overlap each other, or whether they overlap the log region of the mean velocity. In this paper, we shall discuss the upper and lower bounds of $p$ and $w$ intensity log profiles, and also the minimum Reynolds number where the log profiles are first observed.

Third, in addition to the logarithmic intensity profile of the second-order moment, Meneveau and Marusic [28] indicate that this logarithmic law is present in general for the even-order $(2 n$ order) moments of the streamwise velocity fluctuations, where $n$ is a positive integer. The higherorder moments of the pressure fluctuations in turbulent channel flows have not been reported in any previous study according to our knowledge, and we discuss them here beside those of the spanwise velocity fluctuations along with their Reynolds number dependency.

To achieve our goals, we employ a DNS database of channel flow with friction Reynolds numbers ranging from 180 to 4000 , based on the channel half depth and the friction velocity. The paper is organized as follows. Section II overviews the utilized DNS datasets in the analysis. The results are discussed in Secs. III-V. In Sec. III, we present the logarithmic behaviors of $\left\langle p^{+^{2}}\right\rangle$ and $\left\langle w^{+^{2}}\right\rangle$ as well as the higher-order moments of $p$ and $w$. Section IV discusses the spectra and correlations of $p$ and $w$ fluctuations and shows the similarities and the differences between the two fluctuating quantities. In Sec. V, we find the coherent structures related to the $p$ and $w$ fields and illustrate the self-similar distance-from-the-wall character of the coherent structures. Finally, in Sec. VI, we present our conclusions.

\section{DATABASE OF TURBULENT CHANNEL FLOW}

For the present numerical investigation, we employ the DNS database [29] of fully developed incompressible turbulent flows between two parallel planes. The incompressible Navier-Stokes equations are spatially discretized by a Fourier spectral method in the wall-parallel directions and via a second-order central difference method in the wall-normal direction [30,31]. The convection, viscous, and pressure terms are time advanced by the third-order accurate Runge-Kutta method, 
TABLE I. Summary of the parameters of the DNS dataset.

\begin{tabular}{lccccccccr}
\hline \hline $\operatorname{Re}_{\tau}$ & $L_{x} / h$ & $L_{y} / h$ & $L_{z} / h$ & $\Delta x^{+}$ & $\Delta y_{w}^{+}$ & $\Delta y_{c}^{+}$ & $\Delta z^{+}$ & $N_{x} \times N_{y} \times N_{z}$ & $T^{+} / \operatorname{Re}_{\tau}$ \\
\hline 180 & 12.8 & 2.0 & 6.4 & 12.0 & 0.3 & 3.8 & 7.2 & $192 \times 192 \times 160$ & 12.4 \\
400 & 12.8 & 2.0 & 6.4 & 13.3 & 0.17 & 4.3 & 6.7 & $384 \times 384 \times 384$ & 10.4 \\
1000 & 12.8 & 2.0 & 6.4 & 13.3 & 0.6 & 8.0 & 8.3 & $960 \times 512 \times 768$ & 11.2 \\
2000 & 16.0 & 2.0 & 6.4 & 16.0 & 0.6 & 8.0 & 8.3 & $2000 \times 1024 \times 1536$ & 13.3 \\
4000 & 16.0 & 2.0 & 6.4 & 16.0 & 0.6 & 8.0 & 8.3 & $4000 \times 2048 \times 3072$ & 14.0 \\
\hline \hline
\end{tabular}

the Crank-Nicolson method, and the implicit Euler method, respectively. Poisson's equation for the pressure is solved by the two-dimensional fast Fourier-transform scheme and a tridiagonal matrix algorithm. The coordinate system is $(x, y, z)$ where $x, y$, and $z$ represent the streamwise, wall-normal, and spanwise coordinates, respectively. The corresponding velocity fluctuations in the three directions are given by $u_{i}$ with $i=1,2,3$ or $(u, v, w)$. The mean velocities in the three directions are expressed as $U_{i}$ with $i=1,2,3$ or $(U, V, W)$. The total velocities are given by $u_{i}^{t}$, which have the mean and the fluctuating parts, and $p^{t}$ is the total pressure. The channel half depth is $h$, and $L_{x}, L_{y}$, and $L_{z}$ denote the computational domain sizes in the streamwise, wall-normal, and spanwise directions, respectively. Details of the DNS database are presented in Table I. The domain size is relatively large for detecting the large-scale structures around the center of the channel. Total time integration lengths $T^{+}$normalized by the Reynolds number $\left(T^{+} / \operatorname{Re}_{\tau}=T u_{\tau} / h\right)$ to obtain the stable statistical results are summarized in Table I. Periodic boundary conditions are applied in $x$ and $z$ directions, and no-slip and no-penetration boundary conditions are imposed on the velocities at the wall. The grid spacing is uniform in the streamwise and spanwise directions and is refined near the wall in the $y$ direction to account for the large velocity gradient there. Hence, a hyperbolic tangent algebraic equation is applied for the grid spacing in the $y$ direction. Table I displays the grid resolutions in the three directions $\left(\Delta x^{+}, \Delta y^{+}, \Delta z^{+}\right)$, the grid resolution at the wall $\Delta y_{w}^{+}$, and the grid resolution at the center of the channel $\Delta y_{c}^{+}$. The number of grid points in each direction is also given in the table. The plus sign + denotes that the variable is scaled in wall units, where the friction velocity $u_{\tau}$ is the velocity scale, and the viscous length $v / u_{\tau}$ represents the length scale. For convenience, throughout the paper, we refer to structures smaller than $0.7 \mathrm{~h}$ as small-scale motions, which will be discussed further in Sec. IV.

\section{MOMENTS OF FLUCTUATING PRESSURE AND SPANWISE VELOCITY}

\section{A. Second-order moment}

The pressure variance is plotted versus the distance from the wall normalized with the outer variable $h$ in Fig. 1(a) for the different Reynolds numbers. The log trend of the pressure variance is clearly observed to follow Eq. (3) within certain wall-normal locations as previously shown by Jiménez and Hoyas [5] and Sillero et al. [12] in channel flows. The values of the coefficients we obtain are $A_{p}=2.511$ and $B_{p}=0.3951$ from fitting the pressure variance to our highest Reynolds number $\operatorname{Re}_{\tau}=4000$ between $y_{p s}^{+} \approx 150$ and $y_{p e}^{+} \approx 1200$, where $y_{p s}^{+}$(starting point) and $y_{p e}^{+}$(end point) indicate the wall-normal locations where the pressure log trend starts and ends, respectively. The value of the coefficient $A_{p}$ agrees well with the relationship suggested by Panton et al. [26], whereas it is slightly different from that introduced by Sillero et al. [12]: $\left\langle p^{+^{2}}\right\rangle=-2.75 \ln (y / h)+$ 0.1. Panton et al. [26] obtained their relation from fitting the DNS dataset of channel flow of Lee and Moser [25] at $\operatorname{Re}_{\tau}=5186$ between $y_{p s}^{+}=200$ and $y_{p e}^{+}=1000$, whereas Sillero et al. [12] obtained the logarithmic fitting from DNS datasets of channel flow up to $\operatorname{Re}_{\tau}=2000$. However, Sillero et al. [12] did not indicate the bounds of the pressure log-trend region. It seems that the value of $A_{p}$ may be sensitive to $y_{p s}^{+}$as all the curves of the pressure variance for the different Reynolds numbers collapse quite well away from the wall, as shown in Fig. 1(a). 


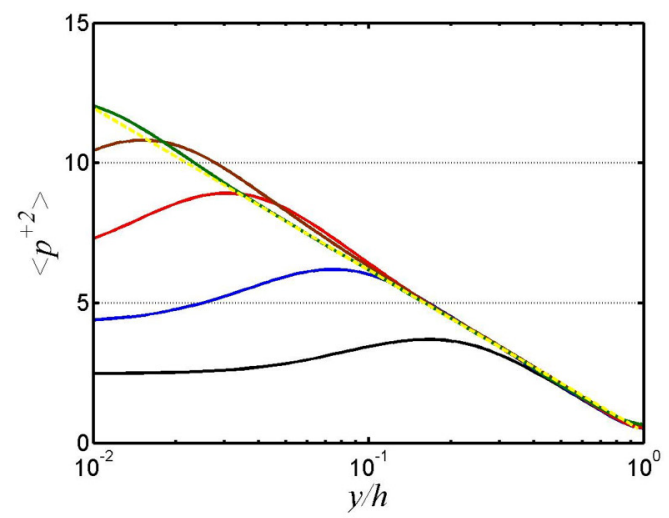

(a)

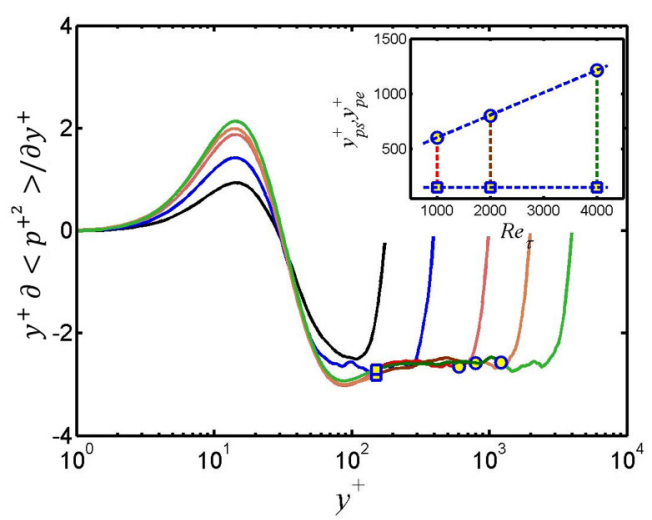

(b)

FIG. 1. (a) Pressure intensity profile in outer variable $y / h$. The dashed yellow line is the logarithmic fit $\left\langle p^{+^{2}}\right\rangle=-A_{p} \ln (y / h)+B_{p}$, with $A_{p}=2.511$ and $B_{p}=0.3951$. (b) Pressure indicator function $\beta_{p p}=$ $y^{+} \partial\left\langle p^{+^{2}}\right\rangle / \partial y^{+}$in inner variable $y^{+}$. Yellow squares and circles represent the wall-normal locations where the pressure intensity log trend starts and ends, $y_{p s}^{+}$and $y_{p e}^{+}$, respectively. $y_{p s}^{+}$and $y_{p e}^{+}$are plotted vs $\operatorname{Re}_{\tau}$ in the inset, where $y_{p s}^{+}=150$ and $y_{p e}^{+}=0.204 \mathrm{Re}_{\tau}+395.6$. In panels (a) and (b), the solid lines represent different Reynolds numbers, $\operatorname{Re}_{\tau}=180(-), \operatorname{Re}_{\tau}=400(-), \operatorname{Re}_{\tau}=1000(-), \operatorname{Re}_{\tau}=2000(-)$, and $\operatorname{Re}_{\tau}=4000(-)$.

Here, for the determination of $y_{p s}^{+}$and $y_{p e}^{+}$of the pressure log trend, the pressure indicator function $\beta_{p p}=y^{+} \partial\left\langle p^{+^{2}}\right\rangle / \partial y^{+}$is employed as the diagnostic tool (e.g., Panton et al. [26]). Figure 1(b) shows $\beta_{p p}$ for different Reynolds numbers, and $\beta_{p p}$ is approximately flat between the starting point $y_{p s}$ and the end point $y_{p e}$. It is also observed that the three Reynolds numbers $\operatorname{Re}_{\tau}=1000,2000$, and 4000 collapse quite well along the plateau region, which indicates that the value of the coefficient $A_{p}$ should be the same irrespective of $\operatorname{Re}_{\tau}$ once the appropriate bounds are determined. Further, we observe from the inset in Fig. 1(b) that when $y_{p s}$ and $y_{p e}$ are plotted against Reynolds number the starting point is independent of Reynolds number, whereas the end point is approximated by a linear function of $\operatorname{Re}_{\tau}$ as $y_{p e}^{+}=0.204 \mathrm{Re}_{\tau}+395.6$. Thus, the inequality $y_{p s} \leqslant y_{p e}$ may provide a sufficient condition for the existence of the log trend of the pressure variance, and the pressure intensity $\log$ region overlaps two regions that scale with the inner and outer variables [5,26]. Although the log trend of the pressure variance is observed for the lower Reynolds number as shown in Fig. 1(a) for $\operatorname{Re}_{\tau}=180$ and 400, the pressure indicator function suggests that it appears unequivocally only when Reynolds number increases, or when the condition $y_{p s} \leqslant y_{p e}$ is realized. It is noted that our DNS datasets were validated by comparing the results of the second-order moments shown in Fig. 1 with those of other DNS databases reported in [5,25], and they were found to be consistent with each other.

Unlike the streamwise velocity fluctuations, the spanwise velocity fluctuations have received less attention in the wall-bounded flow studies. The log behavior in $\left\langle w^{+^{2}}\right\rangle$ was first suggested by Townsend [1] and confirmed using DNS [5,12,23-26], as depicted in Fig. 2(a). The coefficients $A_{w}$ and $B_{w}$ are evaluated within the region bounded by $y_{w s}^{+} \approx 90$ and $y_{w e}^{+} \approx 800$ at $\operatorname{Re}_{\tau}=4000$. The values of $A_{w}=0.4048$ and $B_{w}=0.99$ from $\operatorname{Re}_{\tau}=4000$ are in good agreement with the results of Lee and Moser [25], who assigned the fit to be $\left\langle w^{+^{2}}\right\rangle=-0.387 \ln (y / h)+1.08$, while our fitting is somewhat different from the one suggested by Sillero et al. [12], $\left\langle w^{+^{2}}\right\rangle=-0.45 \ln (y / h)+0.8$, and by Jiménez [24], $\left\langle w^{+^{2}}\right\rangle=-0.45 \ln (y / h)+0.9$, and they also did not state the bounds of the fitting. The slight differences in the fitting could be explained from Fig. 2(b), that shows the indicator function $\beta_{w w}=y^{+} \partial\left\langle w^{+^{2}}\right\rangle / \partial y^{+}$of the spanwise velocity. $\beta_{w w}$ displays an obvious plateau for Reynolds number starting from $\operatorname{Re}_{\tau}=1000$ between $y_{w s}^{+} \approx 90$ and $y_{w e}^{+} \approx 200$. These bounds match the ones stated by Lee and Moser [25] at the same Reynolds number. 


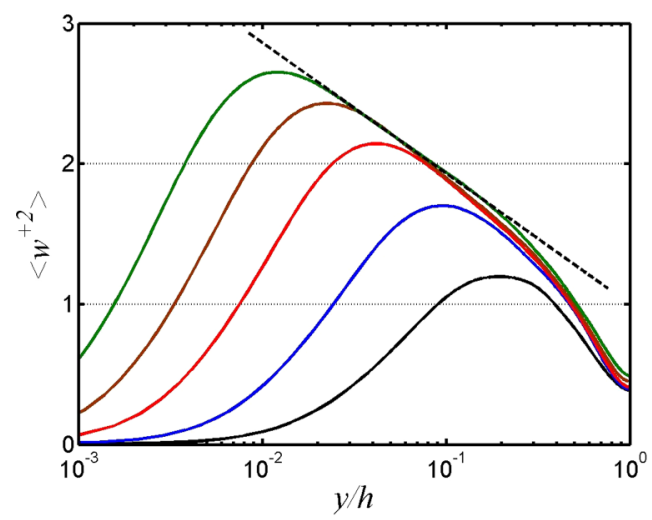

(a)

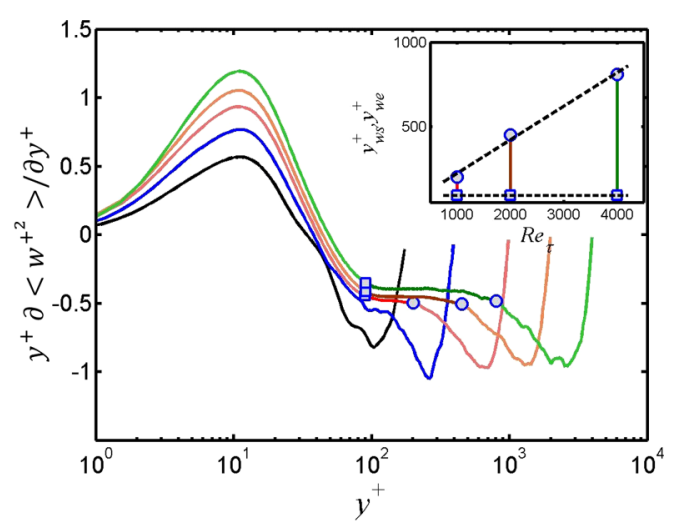

(b)

FIG. 2. (a) Spanwise velocity intensity profile in outer variable $y / h$. The dashed black line is the logarithmic fit $\left\langle w^{+^{2}}\right\rangle=-A_{w} \ln (y / h)+B_{w}$, with $A_{w}=0.4048$ and $B_{w}=0.99$. (b) Spanwise velocity indicator function $\beta_{w w}=y^{+} \partial\left\langle w^{+^{2}}\right\rangle / \partial y^{+}$in inner variable $y^{+}$. Gray squares and circles represent the wall-normal locations where the spanwise velocity intensity log trend starts and ends, $y_{w s}^{+}$and $y_{w e}^{+}$, respectively. $y_{w s}^{+}$and $y_{w e}^{+}$are plotted vs $\operatorname{Re}_{\tau}$ in the inset, where $y_{w s}^{+}=90$ and $y_{w e}^{+}=0.2 \mathrm{Re}_{\tau}+22.3$. In panels (a) and (b), the solid lines represent different Reynolds numbers, $\operatorname{Re}_{\tau}=180(-), \operatorname{Re}_{\tau}=400(-), \operatorname{Re}_{\tau}=1000(-), \operatorname{Re}_{\tau}=2000(-)$, and $\operatorname{Re}_{\tau}=4000(-)$.

Unlike $\beta_{p p}$ displayed in Fig. 1(b), the plateau region of $\beta_{w w}$ does not collapse very well within our range of Reynolds numbers. It is apparent from Fig. 2(b) that $A_{w}$ associated with the plateau of $\beta_{w w}$ changes slightly from around 0.4855 at $\operatorname{Re}_{\tau}=1000$ to $A_{w}=0.4048$ at $\operatorname{Re}_{\tau}=4000$. This trend can also be observed in the study of Lee and Moser [25] [see Fig. 6(c) in Lee and Moser [25]] although they did not refer to it. However, the agreement between our fit and that of Lee and Moser [25] suggests that a collapse for $\beta_{w w}$ would be achieved as Reynolds number increases. On the other hand, the difference between our fit and that of Sillero et al. [12] could be attributed to two reasons. The first reason is the bounds of the $\log$ region of $\left\langle w^{+^{2}}\right\rangle$, which were not stated by Sillero et al. [12], and the second one is their slightly lower Reynolds number of $\operatorname{Re}_{\tau}=2000$.

Furthermore, similar to the bounds of the variance of $p, y_{w s}^{+}$does not depend on Reynolds number, whereas $y_{w e}^{+}$follows a linear relation with $\operatorname{Re}_{\tau}$ as $y_{w e}^{+}=0.2 \operatorname{Re}_{\tau}+22.3$, as indicated in the inset in Fig. 2(b). In contrast to the variance of $w$, the variance of $u$ does not show this log trend at the same Reynolds numbers as reported in a previous study [25], and the results are not shown here for brevity. The turbulent eddies associated with the $\left\langle w^{+^{2}}\right\rangle$ log trend have length scales smaller than those responsible for the $\left\langle u^{+^{2}}\right\rangle \log$ trend [5,27], and this could be one reason for the log trend in $\left\langle w^{+^{2}}\right\rangle$ to be observed at lower Reynolds number.

An additional point can be observed from the insets in Figs. 1(b) and 2(b) and is further elaborated in Appendix A. This additional point is related to the fact that $\left\langle p^{+^{2}}\right\rangle$ exhibits a log trend that has lightly longer wall-normal extent than $\left\langle w^{+^{2}}\right\rangle$, even though $\left\langle p^{+^{2}}\right\rangle$ starts its log behavior at a slightly higher wall-normal location than $\left\langle w^{+^{2}}\right\rangle$. Also explained in Appendix A is that we require a minimum of $\operatorname{Re}_{\tau}=500$ to observe the log trend in both $\left\langle p^{+^{2}}\right\rangle$ and $\left\langle w^{+^{2}}\right\rangle$.

\section{B. Higher-order moments}

The log trend of $\left\langle u^{+^{2}}\right\rangle$ (the second-order moment) was extended by Meneveau and Marusic [28] to higher-order moments $\left\langle u^{2^{2 n}}\right\rangle^{1 / n}$, where $n$ is a positive integer. They found that the $n$th root of the even-order ( $2 n$-order) moments of $u$ follows a generalized logarithmic law with slopes $A_{u_{n}}$ :

$$
\left\langle u^{+^{2 n}}\right\rangle^{1 / n}=-A_{u_{n}} \ln (y / h)+B_{u_{n}} .
$$




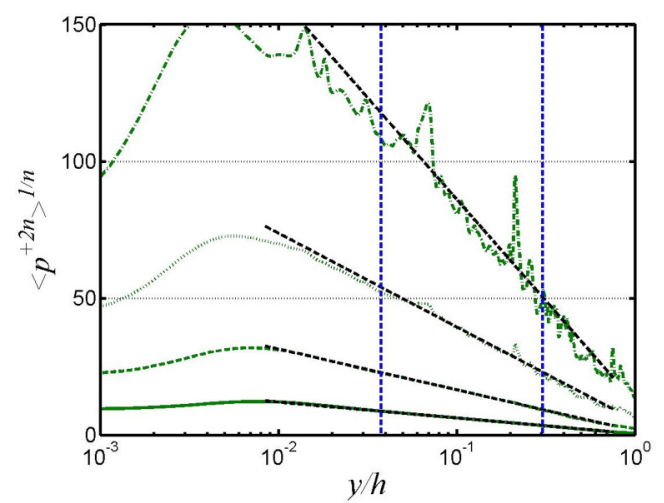

(a)

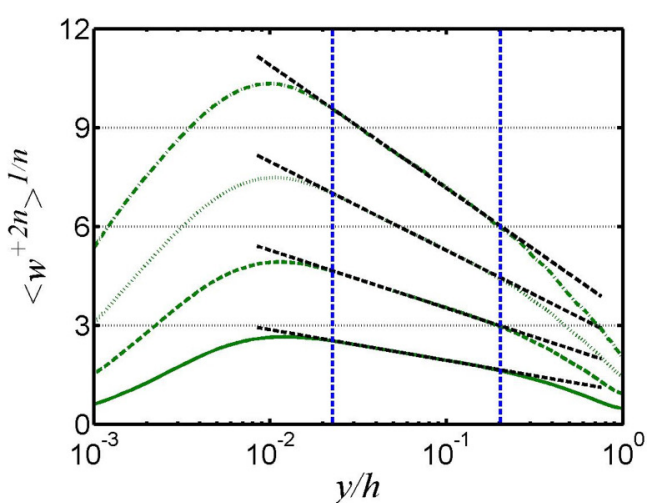

(b)

FIG. 3. $2 n$-order moments raised to the power of $1 / n$ (a) for the pressure fluctuations $\left\langle p^{+^{2 n}}\right\rangle^{1 / n}$, and (b) spanwise velocity fluctuations $\left\langle w^{+^{2 n}}\right\rangle^{1 / n}$, in outer variable $y / h$ at $\operatorname{Re}_{\tau}=4000$ with $n=1(-), n=2(-), n=3$ ( $\cdots$ ), and $n=4$ (-.). The black dashed lines show in panel (a) the fittings $\left\langle p^{+^{2 n}}\right\rangle^{1 / n}=-A_{p_{n}} \ln (y / h)+B_{p_{n}}$, with $A_{p_{n}}=2.511,6.47,14.87,32.26$ and $B_{p_{n}}=0.3951,1.51,5.182,11.85$, and in panel (b) the fittings $\left\langle w^{+^{2 n}}\right\rangle^{1 / n}=$ $-A_{w_{n}} \ln (y / h)+B_{w_{n}}, A_{w_{n}}=0.4048,0.7621,1.169,1.622$, and $B_{w_{n}}=0.99,1.768,2.576,3.422$ for $n=1,2,3$ and 4 , respectively. The blue vertical dashed lines are corresponding to $y_{p s} / h$ and $y_{p e} / h$ in panel (a), and $y_{w s} / h$ and $y_{w e} / h$ in panel (b).

They stated that the slopes $A_{u_{n}}$ are insensitive to the Reynolds number indicating the existence of a universal behavior for $2 n$-order moments of $u$ in wall-bounded turbulence. More recently, Xia et al. [32] confirmed the same behavior for the even-order moments of $u$ in a significant part of a turbulent channel flow with a spanwise rotation.

Meneveau and Marusic [28] explained this tendency from the attached eddy model. They indicated that the streamwise velocity fluctuations at some distance from the wall consist of the sum of independent velocity increments. These velocity increments are associated with a hierarchical organization of self-similar wall-attached eddies. The summands of the velocity increments are statistically independent, which means that the attached eddies do not interact with each other. The consequence of this statistical independence among the summands is the tendency of the Gaussian behavior and for the even-order moments of the streamwise velocity fluctuations to behave like $\left\langle u^{+^{2 n}}\right\rangle \rightarrow(2 n-1) ! !\left\langle u^{+^{2}}\right\rangle^{n}$, where $n ! !=n(n-2)(n-4) \ldots 2$ is the double factorial. A linear model based on this simplification of the attached eddy model was developed by Yang et al. [33]. The behavior of the spanwise velocity fluctuations is predicted from the attached model, and hence their higher-order moments are expected to show a logarithmic behavior. Therefore, they are related to a hierarchical organization of eddies at smaller length scales than those of $u$, as will be clarified in the next section. Interestingly, for the pressure fluctuations also, the log behaviors of the higher-order moments can be observed [see Fig. 3(a)], and as such a relation between $p$ and the self-similar velocity-based eddies could be expected. Specifically, Figs. 3(a) and 3(b) show the profiles of $\left\langle p^{+^{2 n}}\right\rangle^{1 / n}$ and $\left\langle w^{+^{2 n}}\right\rangle^{1 / n}$ for $n=1,2,3$, and 4 at $\operatorname{Re}_{\tau}=4000$ plotted against $y / h$. The data indeed show logarithmic behaviors for $\left\langle p^{+^{2 n}}\right\rangle^{1 / n}$ and $\left\langle w^{+^{2 n}}\right\rangle^{1 / n}$ represented as

$$
\begin{gathered}
\left\langle p^{+^{2 n}}\right\rangle^{1 / n}=-A_{p_{n}} \ln (y / h)+B_{p_{n}}, \\
\left\langle w^{2^{2 n}}\right\rangle^{1 / n}=-A_{w_{n}} \ln (y / h)+B_{w_{n}} .
\end{gathered}
$$

It is observed that the higher-order moments show the log trend between $y_{p s}$ and $y_{p e}$ for the pressure, and between $y_{w s}$ and $y_{w e}$ for the spanwise velocity. We may note that the higher-order moments of the pressure fluctuations are less statistically converged (especially for $n=4$ ) compared 


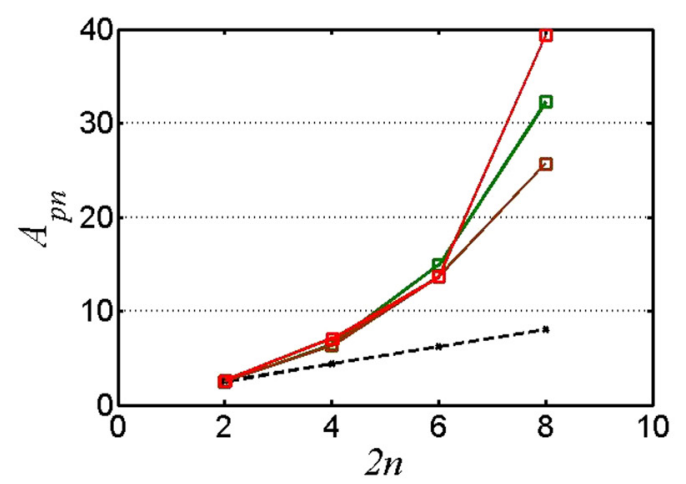

(a)

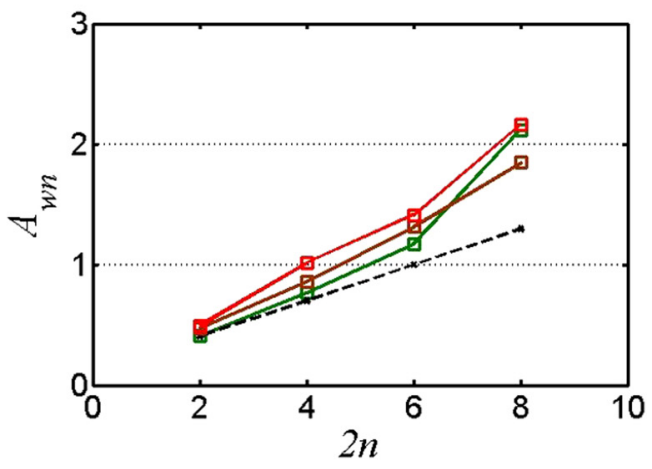

(b)

FIG. 4. The slopes (a) $A_{p_{n}}$ and (b) $A_{w_{n}}$ as functions of the moment order $2 n$ at $\operatorname{Re}_{\tau}=1000(-), \operatorname{Re}_{\tau}=2000$ $(-)$, and $\operatorname{Re}_{\tau}=4000(-)$. The black dashed line represents the Gaussian behavior $A_{p_{n}}=A_{p_{1}}[(2 n-1) ! !]^{1 / n}$ in panel (a), and $A_{w_{n}}=A_{w_{1}}[(2 n-1) ! !]^{1 / n}$ in panel (b).

with those of the spanwise velocity fluctuations. For the case of streamwise velocity, Meneveau and Marusic [28] discussed the even-order moments up to $n=5$ for turbulent boundary layers, and Xia et al. [32] presented the analysis up to $n=6$ in a turbulent channel flow with a spanwise rotation. The main reason for the difficulty in convergence is because the pressure fluctuations are of the order of the square of the velocity fluctuations, $\mathcal{O}\left(u^{2}\right)$, and therefore it is difficult to get good statistically converged results beyond $n=3$ for the pressure fluctuations. The error in the log fitting of Eqs. (5) and (6) to the data and an investigation of the statistical convergence using premultiplied probability density functions (PDFs) are presented in Appendix B.

In order to explore the Reynolds number dependence of the slopes $A_{p_{n}}$ and $A_{w_{n}}, 2 n$-order moments were fitted up to $n=4$ at $\operatorname{Re}_{\tau}=1000,2000$, and 4000 between $y_{p s}^{+}$and $y_{p e}^{+}$for the pressure, and within the range $\left[y_{w s}^{+}, y_{w e}^{+}\right]$for the spanwise velocity. The slopes are plotted as a function of Reynolds number in Fig. 4 as square symbols. As shown by Meneveau and Marusic [28] for streamwise velocity, if the pressure fluctuations follow a Gaussian behavior, then $A_{p_{n}}=$ $A_{p_{1}}[(2 n-1) ! !]^{1 / n}$, where $A_{p_{1}}=A_{p}$ in Eq. (3). The Gaussian behavior of the slopes $A_{p_{n}}$ is presented in Fig. 4(a) as a dashed black line. Clearly, unlike the slopes $A_{u_{n}}$ of the streamwise velocity fluctuations that show a "sub-Gaussian" behavior, $A_{p_{n}}$ seem to follow a "super-Gaussian" one. This behavior may be expected as the flatness of the pressure fluctuations across the channel is higher than the Gaussian value of 3, independent of Reynolds number (results of the flatness are not shown here for brevity). Also, from Fig. 4(a) we could discern that $A_{p_{n}}$ does not depend on Reynolds number. The slopes $A_{w_{n}}$ shown in Fig. 4(b) also display the super-Gaussian behavior for the different Reynolds numbers. However, the tendency towards the Gaussian behavior is asymptotically approached as Reynolds number increases.

In conclusion, the log trend of the second-order moment in addition to the higher-order moments of pressure fluctuations have been presented along with those of the spanwise velocity fluctuations. The pressure and spanwise velocity fluctuations show their logarithmic trends at lower Reynolds numbers compared with the streamwise velocity fluctuations. In the next section, we attempt to explore the structures in spectral and physical spaces related to these log profiles of $\left\langle p^{+^{2}}\right\rangle$ and $\left\langle w^{+^{2}}\right\rangle$.

\section{SPECTRA AND CORRELATIONS OF $p$ AND $w$}

To explore the structures related to $\left\langle p^{+^{2}}\right\rangle$ and $\left\langle w^{+^{2}}\right\rangle \log$ profiles, we first look at the spectra as they are directly related to the variance. Even though they were discussed extensively in previous studies [5,27], this will serve as a basis for much of our discussion in coming sections. 


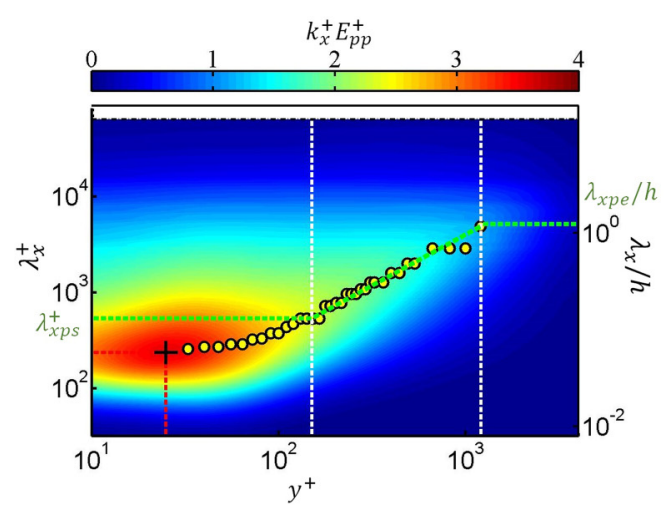

(a)

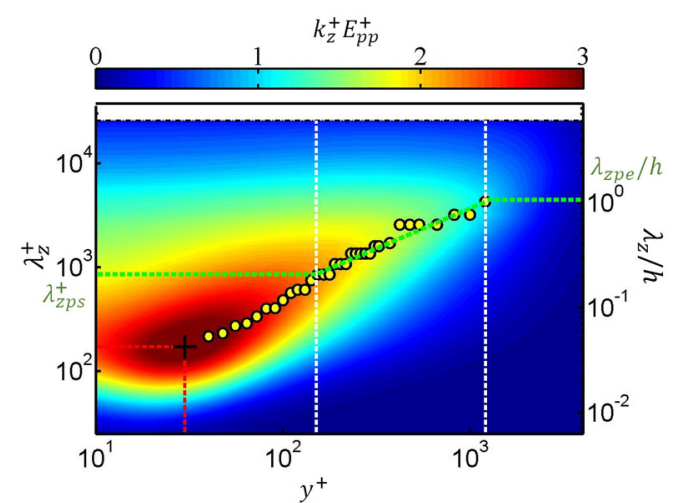

(b)

FIG. 5. Premultiplied spectra in (a) streamwise direction $k_{x} E_{p p}\left(k_{x}\right) / \rho^{2} u_{\tau}^{4}$, and (b) spanwise direction $k_{z} E_{p p}\left(k_{z}\right) / \rho^{2} u_{\tau}^{4}$ vs $y^{+}$and wavelengths at $\operatorname{Re}_{\tau}=4000$. Yellow circles highlight the local peaks of the spectra at each wall-normal height, and the plus mark highlights the peak of the spectra. In each panel, the two white vertical dashed lines indicate $y_{p s}^{+}$and $y_{p e}^{+}$and the two dashed horizontal green lines represent $\lambda_{x p s}^{+}$and $\lambda_{x p e} / h$ in panel (a), and $\lambda_{z p s}^{+}$and $\lambda_{z p e} / h$ in panel (b). The dashed green line connects the two points $\left(\lambda_{x p s}^{+}, y_{p s}^{+}\right)$and $\left(\lambda_{x p e} / h, y_{p e}^{+}\right)$in panel (a), and the two points $\left(\lambda_{z p s}^{+}, y_{p s}^{+}\right)$and $\left(\lambda_{z p e} / h, y_{p e}^{+}\right)$in panel (b).

\section{A. Pressure spectra}

Figure 5(a) shows the contour plots of the premultiplied one-dimensional (1D) spectra of the pressure fluctuations, $k_{x}^{+} E_{p p}^{+}=k_{x} E_{p p}\left(k_{x}\right) / \rho^{2} u_{\tau}^{4}$, at $\operatorname{Re}_{\tau}=4000$, where $E_{p p}\left(k_{x}\right)$ are the pressure spectra in the streamwise direction and $k_{x}$ is the streamwise wave number. Following Jiménez and Hoyas [5], in the figure, contours of $k_{x}^{+} E_{p p}^{+}$are plotted versus the distance from the wall $y^{+}$and the streamwise wavelength $\lambda_{x}^{+}$. The axes are represented on logarithmic scales. The white dashed vertical lines represent the boundaries of the pressure log region, $y_{p s}^{+}$and $y_{p e}^{+}$. The black plus mark in the plot indicates the peak of the spectra that occurs at a fixed wall-normal height of $y^{+} \approx 25$ associated with a wavelength equal to approximately 240 in viscous units, $\left(y^{+}, \lambda_{x}^{+}\right)_{\text {peak }} \approx(25,240)$, irrespective of the Reynolds number. Therefore, the contribution to the peak comes from the length scales that are scaled in wall units. In addition, it is noted that the position of the peak of the spectra matches the peak position of the pressure variance shown in Fig. 1(a) that occurs at $y^{+} \approx 30$ and does not also depend on $\operatorname{Re}_{\tau}$. This was also reported previously by Kim [34] (albeit at lower $\operatorname{Re}_{\tau}$ ) and Tsuji et al. [10] for turbulent boundary layers.

Tsuji et al. [6] carried out the measurements for the static pressure at different wall-normal locations within the turbulent boundary layer for a wide range of Reynolds number. From the spectral analysis of the pressure fluctuations upon applying Taylor's hypothesis, they observed the existence of a second peak in the premultiplied spectra. The results of the pressure premultiplied spectra were shown for $\operatorname{Re}_{\tau}=3583$ and 6890. However, they did not report whether the second peak exists for Reynolds numbers lower than $\operatorname{Re}_{\tau}=3583$. The second peak was located at $y / \delta \approx 0.1$, and it was associated with a wavelength of $\lambda_{x}^{+} \approx 4400$ for $\operatorname{Re}_{\tau}=6890$. For its location at $y / \delta \approx 0.1$, the second peak was referred to as the "outer" peak. In our results of channel flow, the outer peak does not exist even for $\operatorname{Re}_{\tau}=4000$. The nonexistence of the outer peak in channel flow is attributed to the difference in the outer region between the boundary layer and channel flows. This was clarified to some extent by Sillero et al. [13] from the discussion of the two-point correlations of the pressure fluctuation in boundary layers and channels. They observed that the pressure correlation in the outer layer of boundary layers extends all the way from the wall to the potential region. However, the correlation in channels reaches across the full half channel. Further, they indicated a deeper negative lobe of the streamwise correlation in boundary layers than in channels, assigning that to the stronger 
pressure fluctuations in the potential flow outside the boundary layer. Recently, Panton et al. [26] also pointed out the difference between the two flow fields when discussing the correlations of the pressure fluctuations in the inner and outer regions.

The yellow circles in Fig. 5(a) represent the local peaks of the spectra at each wall-normal height. Jiménez and Hoyas [5] and Baidya et al. [35] referred to these peaks as the "ridge" of the 1D spectra, and they characterized the dominant streamwise length scale at a definite location from the wall $[25,36]$. Results in the previous section suggest that it is important to look at the ridge of the spectra between $y_{p s}^{+}$and $y_{p e}^{+}$. From Fig. 5(a), it is observed that the ridge of the spectra at $y_{p s}^{+}$ is associated with a streamwise wavelength of $\lambda_{x p s}^{+} \approx 450$ for all Reynolds numbers. On the other hand, the streamwise wavelength associated with the ridge of the spectra at $y_{p e}^{+}$is $\lambda_{x p e} / h \approx 1$, and this value also does not depend on $\operatorname{Re}_{\tau}$. What we could infer from this is that the streamwise length scale of the dominant structures associated with the pressure log profile identified between $y_{p s}^{+}$and $y_{p e}^{+}$overlaps over both inner and outer normalizations, as indicated by Jiménez and Hoyas [5] and Jiménez [27]. Further, the largest streamwise length scale of these dominant structures is of the order of the channel half depth. Finally, the dashed green line shown in Fig. 5(a) represents the connection between the two points $\left(\lambda_{x p s}^{+}, y_{p s}^{+}\right)$and $\left(\lambda_{x p e} / h, y_{p e}^{+}\right)$, and exhibits a linear relation between $\lambda_{x}$ and $y$. As could be noticed, the ridge of the spectra at each $y$ between $y_{p s}^{+}$and $y_{p e}^{+}$is consistent with this linear relation. The slope of this linear trend is about 3.8 at our $\operatorname{Re}_{\tau}=4000$, which is somewhat higher than the slope stated by Jiménez and Hoyas [5] that is around 3 at $\operatorname{Re}_{\tau}=2000$. The difference between the two values is due to two reasons. First, this linear trend in our results represents just a connection between the two points $\left(\lambda_{x p s}^{+}, y_{p s}^{+}\right)$and $\left(\lambda_{x p e} / h, y_{p e}^{+}\right)$, and does not represent a linear fit for the ridge of the spectra located between $y_{p s}^{+}$and $y_{p e}^{+}$[5]. Second, Jiménez and Hoyas [5] identified the logarithmic region for the pressure fluctuations to be located between $y^{+} \approx 100$ and $y / h=0.2$, like those of the velocity fluctuations, which are different from our bounds $y_{p s}^{+}$and $y_{p e}^{+}$.

It is worth reporting that the same spectral features of the pressure fluctuations have been confirmed from the recent modeling efforts of Luhar et al. [37] for pipe flow in terms of the resolvent analysis at $\operatorname{Re}_{\tau}=2000$. A linear model was suggested based on the first singular response mode that was found to dominate the velocity field at a definite wavenumber-frequency combination. The pressure spectra established from the linear model confirmed the linear relation between $\lambda_{x}$ and $y$ within the $\log$ region identified between $y^{+} \approx 100$ and $y / h=0.2$. However, they indicated that this linear relation extends in the near-wall and outer regions with different slopes. This extended linear relation is in contrast to our results and to the results of Jiménez and Hoyas [5] where the ridge of the spectra terminates and roughly remains at a constant wavelength close to the wall as shown in Fig. 5(a). For these differences in the near-wall region, the inner peak they reported was found to be located at $y^{+}<60$ and associated with a streamwise wavelength of around $\lambda_{x}^{+}=180$ lower than the value reported previously.

The premultiplied spectra in spanwise direction $k_{z}^{+} E_{p p}^{+}=k_{z} E_{p p}\left(k_{z}\right) / \rho^{2} u_{\tau}^{4}$, plotted in Fig. 5(b) versus $y^{+}$and $\lambda_{z}^{+}$at $\operatorname{Re}_{\tau}=4000$, show the same tendency as the other Reynolds numbers. Here, $k_{z}$ and $\lambda_{z}$ are the spanwise wave number and wavelength, respectively. As in the case of the streamwise spectra, only the inner peak appears. Its location is found at $y_{\text {peak }}^{+} \approx 30$, and does not depend on $\operatorname{Re}_{\tau}$. This location perfectly overlaps the peak position of the pressure variance $\left\langle p^{+^{2}}\right\rangle$ shown in Fig. 1(a). The inner peak of $k_{z}^{+} E_{p p}^{+}$is associated with a spanwise length scale of $\left(\lambda_{z}^{+}\right)_{\text {peak }} \approx 170$ that is smaller than that associated with the inner peak of $k_{x}^{+} E_{p p}^{+}$. The spanwise wavelength associated with the ridge of the spectra at $y_{p e}^{+}$is $\lambda_{z p e} / h \approx 1$ for all $\operatorname{Re}_{\tau}$, while, at $y_{p s}^{+}, \lambda_{z p s}^{+}$slightly changes from around 600 to 900 for $\operatorname{Re}_{\tau}=1000$ to 4000, respectively. Accordingly, it is concluded that the spanwise length scale of the dominant structures associated with the pressure log behavior is $\mathcal{O}(h)$. In addition, the ridge of the spectra between $y_{p s}^{+}$and $y_{p e}^{+}$is located along the line that connects the two points $\left(\lambda_{z p s}^{+}, y_{p s}^{+}\right)$and $\left(\lambda_{z p e} / h, y_{p e}^{+}\right)$. This line has a slope of approximately 3.2 which agrees with the value of 3 reported by Jiménez and Hoyas [5]. In addition, this confirms the fact that the dominant structures of the pressure fluctuations have an approximately equal distribution in streamwise and spanwise directions [5]. 


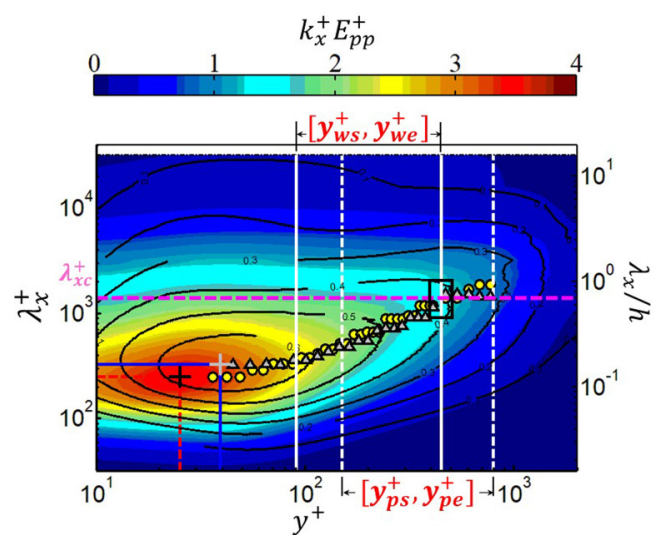

(a)

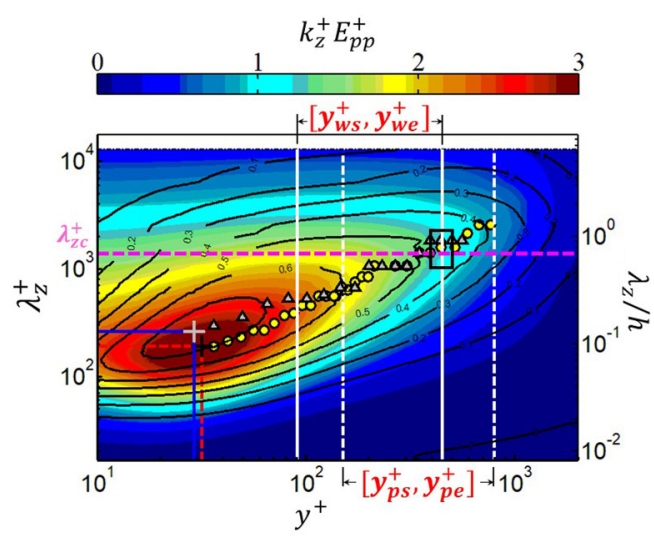

(b)

FIG. 6. Premultiplied spectra in (a) streamwise direction, and (b) spanwise direction of $p$ and $w$ vs $y^{+}$and wavelengths at $\operatorname{Re}_{\tau}=2000$. Yellow circles highlight the local peaks of $p$ spectra at each wall-normal height, and the black plus marker highlights the peak of $p$ spectra. Gray triangles highlight the local peaks of $w$ spectra at each wall-normal height, and the gray plus marker highlights the peak of $w$ spectra. White vertical dashed lines indicate $y_{p s}^{+}$and $y_{p e}^{+}$, while the solid ones represent $y_{w s}^{+}$and $y_{w e}^{+}$. The magenta horizontal line in each panel indicates the cutoff wavelengths $\lambda_{x c}^{+}$and $\lambda_{z c}^{+}$in panels (a) and (b), respectively.

\section{B. Comparison between $p$ and $w$ spectra}

For comparison with the spanwise velocity fluctuations, we show the premultiplied spectra of the spanwise velocity fluctuations $k_{x}^{+} E_{w w}^{+}=k_{x} E_{w w}\left(k_{x}\right) / u_{\tau}^{2}$ in the same plot with those of the pressure fluctuations at $\operatorname{Re}_{\tau}=2000$. Figure 6(a) plots the unfilled contour lines of the premultiplied spectra $k_{x}^{+} E_{w w}^{+}$in conjunction with $k_{x}^{+} E_{p p}^{+}$which are represented by the filled contour lines as in Fig. 5(a). The local peaks of $k_{x}^{+} E_{w w}^{+}$are presented in gray triangular marks, while those of $k_{x}^{+} E_{p p}^{+}$are denoted by yellow circles. Two white dashed vertical lines are also shown that represent $y_{p s}^{+}$and $y_{p e}^{+}$, while the solid ones indicate $y_{w s}^{+}$and $y_{w e}^{+}$. From the figure, we can observe the following. First, for $k_{x}^{+} E_{w w}^{+}$, only the first or the inner peak appears. It is located at $y_{\text {peak }}^{+} \approx 40$ which is apparently close to that of $k_{x}^{+} E_{p p}^{+}$. Second, we discern that the contour lines of $k_{x}^{+} E_{w w}^{+}$overlap those of $k_{x}^{+} E_{p p}^{+}$at some distances across the channel. Third, and more importantly, the dominant streamwise length scales of both $p$ and $w$, indicated by the yellow circles and gray triangles, respectively, are approximately the same (which was also reported by Jiménez and Hoyas [5] and Jiménez [27]) especially in the region extended from $y_{w s}^{+}$to $y_{p e}^{+}$. Furthermore, the results of the spanwise spectra are presented in Fig. 6(b). The dominant spanwise length scales of $p$ and $w$ match with each other between $y_{w s}^{+}$and $y_{p e}^{+}$, but not like those of the streamwise spectra, and the contour lines of the two spectra do not perfectly overlap each other.

The comparison between the premultiplied spectra of $p$ and $w$ empirically supports the similarity between $p$ and $w$ at some distances from the wall, and this leads to the log behaviors reported in Sec. III A. The results also suggest that the dominant turbulent structures associated with the log behaviors of $p$ and $w$ are $\mathcal{O}(h)$ or less in both streamwise and spanwise directions. To clarify the contributions from large and small scales of motions, in the following, we carry out a spectral filter on $p$ and $w$ fields.

\section{Large- and small-scale contributions to $\left\langle\boldsymbol{p}^{\mathbf{+}^{2}}\right\rangle$ and $\left\langle w^{\mathbf{+}^{2}}\right\rangle$}

Here, we split the instantaneous pressure and spanwise velocity fields into large-scale $p_{l}$ and $w_{l}$, and small-scale $p_{s}$ and $w_{s}$. The filtering process is carried out in streamwise and spanwise directions with cutoff wavelengths $\lambda_{x c}=\lambda_{z c}=0.7 h$ for all Reynolds numbers. The cutoff wavelengths are 


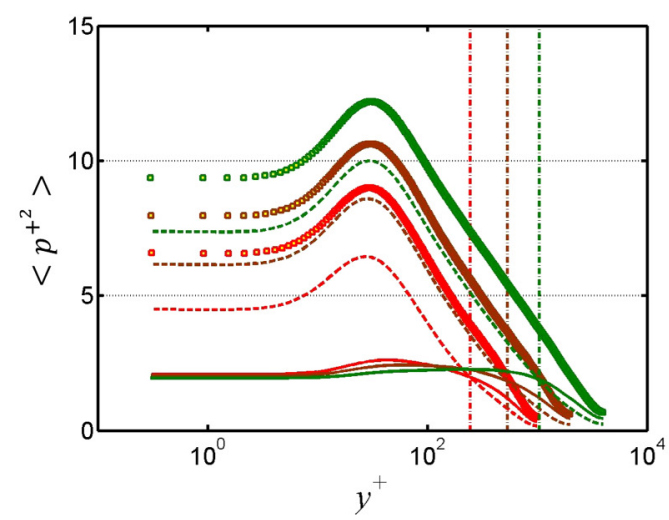

(a)

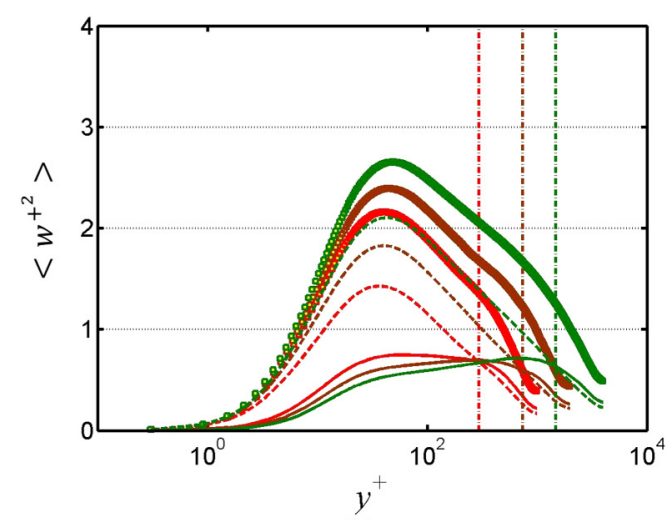

(b)

FIG. 7. (a) Pressure, and (b) spanwise velocity intensity profiles in inner variable $y^{+}$at $\operatorname{Re}_{\tau}=1000$ (red), $\operatorname{Re}_{\tau}=2000$ (brown), and $\operatorname{Re}_{\tau}=4000$ (green). Original profiles are plotted by square symbols. The contributions from small and large scales are plotted by dashed and solid lines, respectively. The vertical dash-dotted lines represent the wall-normal location where the small and large scales intersect at each $\operatorname{Re}_{\tau}$ for the pressure in panel (a), $y_{p s l}^{+}$, and for the spanwise velocity in panel (b), $y_{w s l}^{+}$. In panel (a), $y_{p s l}^{+} \approx 245,535$, and 1050 , and in panel (b) $y_{w s l}^{+} \approx 295,740$, and 1470 for $\operatorname{Re}_{\tau}=1000,2000$, and 4000, respectively.

taken at this value based on the following reasoning. We return to Fig. 6 for the premultiplied 1D spectra of $p$ and $w$ where the filtering wavelengths $\left(\lambda^{+}=0.7 \mathrm{Re}_{\tau}\right)$ are represented in each panel with a magenta horizontal line. We intend to apply the same filter for both $p$ and $w$, and so we select $\lambda_{x c}^{+}$that matches both. Because $y_{w e}^{+}<y_{p e}^{+}$, we select $\lambda_{x c}^{+}$that represents the wavelength which is associated with the ridge of the spectra of either $p$ or $w$ at $y_{w e}^{+}$. From Fig. 6(a), $\lambda_{x c}^{+}=1400\left(\lambda_{x c} / h=0.7\right)$ seems to be a reasonable selection at $\operatorname{Re}_{\tau}=2000$. On the other hand, as shown in Fig. 6(b), $\lambda_{z c}^{+}$seems to be lower than the spanwise wavelength associated with the ridge of the spectra of $p$ and $w$ at $y_{w e}^{+}$. However, $\lambda_{z c}^{+}$is taken equal to $\lambda_{x c}^{+}$as the 2D spectra of $p$ and $w$ are aligned along the line $\lambda_{z}=\lambda_{x}$. Other Reynolds numbers show the same results as those reported in Fig. 6. It is noted that the value of the spectral filter of $0.7 \mathrm{~h}$ can be predicted approximately from the linear relationships of the ridge of the spectra $\lambda_{x} \approx \lambda_{z} \approx 3 y$ presented by Jiménez and Hoyas [5]. They defined the logarithmic region of the pressure to be the same as that of the velocity fluctuations, which extended up to $y / h=0.2$. Their upper bound yields a filter of around $0.6 h$, which agrees with ours of $0.7 h$.

The profiles of the variance of the large- and small-scale pressure and spanwise velocity fluctuations are displayed in Figs. 7(a) and 7(b), respectively. The dashed lines represent the variance of the small scales, while the large scales are represented by the solid ones. The panels also contain the unfiltered fields of $p$ and $w$ which are indicated by the square symbols. It can be seen clearly from the figure that the logarithmic relations of the variances of $p$ and $w$ are confirmed for the scales of motions smaller than $0.7 \mathrm{~h}$. These results of the pressure agree with those of Tsuji et al. [6] for the pressure fluctuations in turbulent boundary layers. A cutoff wavelength of $\lambda_{x c}=\delta / 5$ was selected based on the observations of the inner and outer peaks, as discussed previously. Our results show the inner peak only. Hence, we did not follow their selection.

Further, our results of the spanwise velocity confirm Fig. 5(b) in Baidya et al. [20] for the variance of the large and small scales of $w$ fluctuations for turbulent boundary layers at high Reynolds number $\left(\operatorname{Re}_{\tau}=3000,6000\right.$, and 10500$) . \lambda_{x c}^{+}$was taken to be 2000 by Baidya et al. [20], irrespective of $\operatorname{Re}_{\tau}$. They did not mention why this cutoff level was selected. However, a second peak started to appear for the premultiplied streamwise spectra of $w$ fluctuations for the high Reynolds number $\operatorname{Re}_{\tau}=$ 10 500. In addition, our results corroborate the discussion of Abe et al. [38]. They utilized a DNS dataset with a "minimal" streamwise flow unit $\left(L_{x}^{+} \approx 400\right)$ to examine the large-scale motion (with 
a streamwise length $2-4 h$ ) in the logarithmic and outer regions in channels. They compared the spanwise velocity intensity profile for this minimal streamwise flow unit with that of the large-scale domain $\left(L_{x}=12.8 h\right)$ at the same Reynolds number. Both confirm clear logarithmic profiles for $\left\langle w^{+^{2}}\right\rangle$. They attributed this tendency to the reduction in the spanwise large scales in the minimal flow units.

The vertical dash-dotted lines in each panel in Fig. 7 represent the distances from the wall where the intensities of the small and large scales intersect at each Reynolds number. We define these wall-normal locations as $y_{p s l}$ and $y_{w s l}$ for the pressure in panel (a) and for the spanwise velocity in panel (b), respectively. $y_{p s l}^{+}$takes the values of approximately 245, 535, and 1050 for $\operatorname{Re}_{\tau}=1000$, 2000, and 4000, respectively, while $y_{w s l}^{+}$is corresponding to 295,740, and 1470 at the same Reynolds numbers. For each Reynolds number, it is noted that $y_{p s l}^{+}$and $y_{w s l}^{+}$are less than $y_{p e}^{+}$. Overall, our results show that small-scale motions contribute significantly to the log trend in $\left\langle p^{+^{2}}\right\rangle$ and $\left\langle w^{+^{2}}\right\rangle$, and removing the large scales increases the region over which the log trend is observed.

\section{Correlation functions of small-scale $\left\langle\boldsymbol{p}^{+^{2}}\right\rangle$ and $\left\langle w^{+^{2}}\right\rangle$ motions}

Before discussing the coherent structures in the next section and to further clarify the relation between $p$ and $w$ fields, we define the correlation function of the filtered pressure and spanwise velocity fluctuations. The autocorrelation function for the small scale of $p$ and $w$, following Jiménez [27], is defined as

$$
c_{\psi_{s} \psi_{s}}(\Delta x, y, \tilde{y}, \Delta z)=\frac{\left\langle\psi_{s}(X) \psi_{s}(\tilde{X})\right\rangle}{\psi_{\mathrm{rms}}(y) \psi_{\mathrm{rms}}(\tilde{y})},
$$

where $\psi$ and $\psi_{s}$ refer, respectively, to the total and small scale of $p$ and $w$ (smaller than $0.7 h$ ). Here, $X=(x, y, z), \Delta x=x-\tilde{x}, \Delta z=z-\tilde{z}$, and $\tilde{X}=(\tilde{x}, \tilde{y}, \tilde{z})$ is the reference position. The local root-mean-square (rms) values of the total $p$ and $w$ are selected for the normalization. The correlations of the small-scale pressure $c_{p_{s} p_{s}}$ and spanwise velocity $c_{w_{s} w_{s}}$ are shown in Figs. 8(a) and 8(b), respectively, for $x-y$ sections and $x$-z sections in Figs. 8(c) and 8(d). In each panel, positive and negative correlations are represented by the black and blue contours, respectively. In Figs. 8(b) and $8(\mathrm{~d})$, the gray patches represent $c_{p_{s} p_{s}}$. They are displayed on the same plots of $c_{w_{s} w_{s}}$ for the purpose of comparison. The reference position is taken at $\tilde{y} / h \approx 0.1$ for $\operatorname{Re}_{\tau}=1000$. The correlations $c_{p_{s} p_{s}}$ and $c_{w_{s} w_{s}}$ show similar features observed for total $p$ and $w$ which were discussed in detail by Sillero et al. [13]; however, here they are correlated at smaller length scales due to the filtering process. A significant point here is that both $c_{p_{s} p_{s}}$ and $c_{w_{s} w_{s}}$ are correlated over the same distances, which is apparent in Fig. 8(d) for the positive $c_{p_{s} p_{s}}$ and $c_{w_{s} w_{s}}$ in $x-z$ sections. For $x-y$ sections in Fig. 8(b), positive $c_{p_{s} p_{s}}$ and $c_{w_{s} w_{s}}$ also have the same length with different inclinations to the wall. This is because $p$ and $w$ fluctuations have different orientations with respect to their common structures. Another noteworthy point regarding Fig. 8(d) is that for the spanwise velocity fluctuations there are two negative blobs $\left(c_{w_{s} w_{s}}=-0.05\right)$ on two sides aligned along the spanwise direction. Interestingly, these two negative correlations are not indicated for the total $w$ fluctuations discussed by Sillero et al. [13].

The cross-correlation function $c_{p_{s} w_{s}}$ of the small-scale pressure-spanwise velocity fluctuations is defined as

$$
c_{p_{s} w_{s}}(\Delta x, y, \tilde{y}, \Delta z)=\frac{\left\langle w_{s}(X) p_{s}(\tilde{X})\right\rangle}{w_{\mathrm{rms}}(y) p_{\mathrm{rms}}(\tilde{y})},
$$

and is shown in Fig. 9 for the same values of $\tilde{y} / h \approx 0.1$ and $\operatorname{Re}_{\tau}=1000$. The small-scale pressure is selected to be fixed at the reference position. For the value of the correlation $c_{p_{s} w_{s}}= \pm 0.08$ shown in Fig. 9(a), the positive and negative correlations are approximately symmetric, and they are 


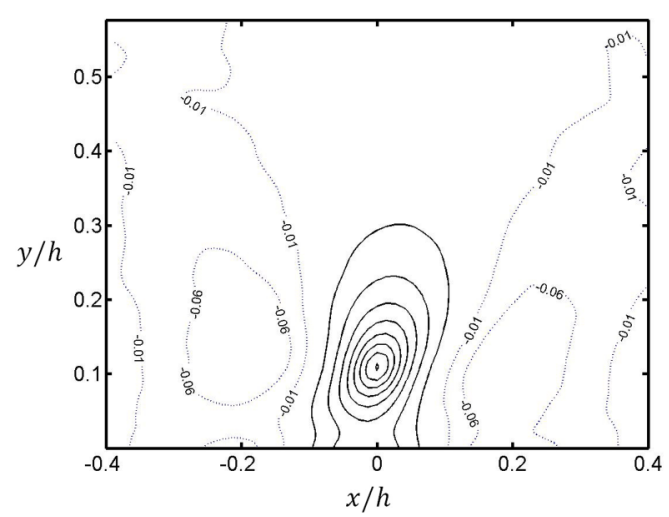

(a)

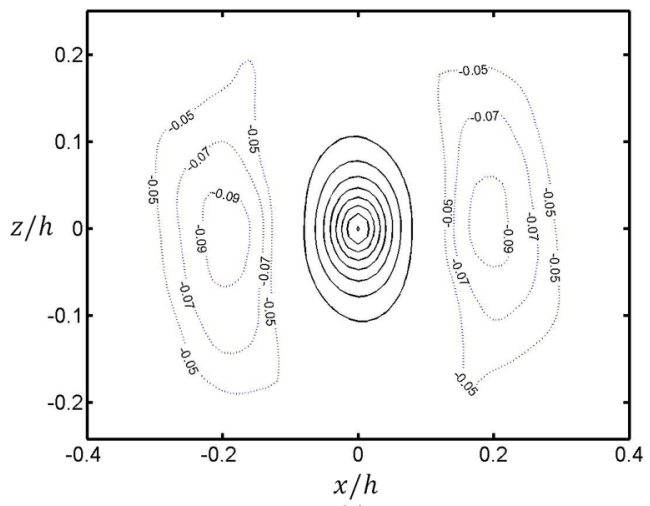

(c)

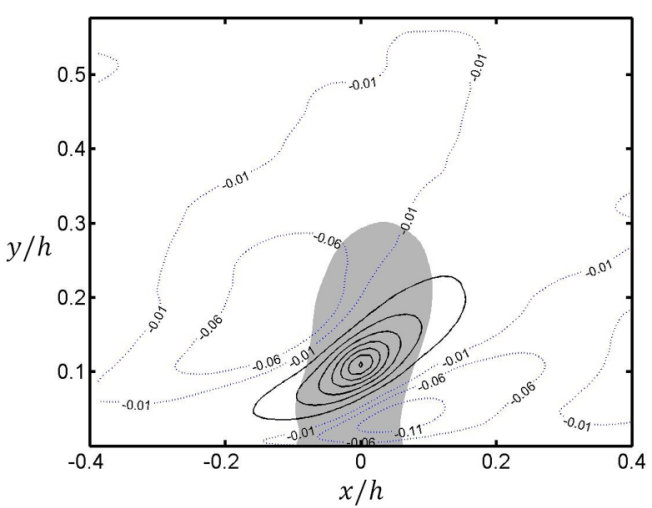

(b)

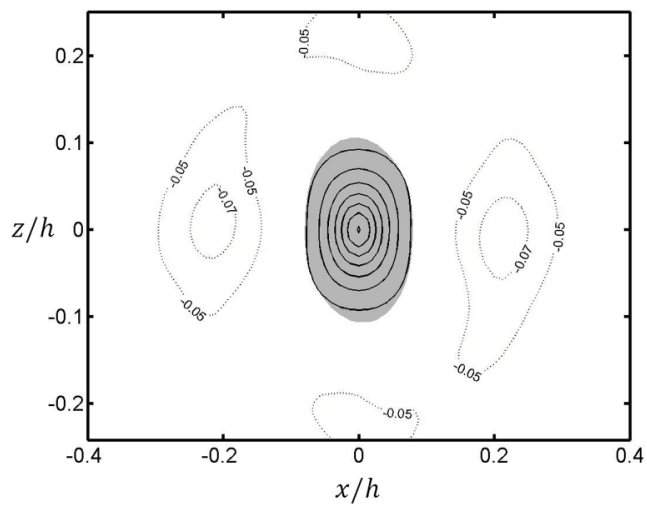

(d)

FIG. 8. Two-dimensional sections through the reference point $(\tilde{y} / h \approx 0.1)$ of the two-point autocorrelation function of the small-scale (a), (c) pressure $c_{p_{s} p_{s}}$, and (b), (d) spanwise velocity $c_{w_{s} w_{s}}$ at $\operatorname{Re}_{\tau}=1000 . x-y$ sections are represented in panels (a) and (b) for $c_{p_{s} p_{s}}$ and $c_{w_{s} w_{s}}$, respectively. $x-z$ sections are introduced in panels (c) and (d) for $c_{p_{s} p_{s}}$ and $c_{w_{s} w_{s}}$, respectively. Positive correlations are indicated by the black color with values [0.1: $0.1: 0.7]$, while the blue dashed contours represent negative correlations. In panels (b) and (d), the gray patches represent $c_{p_{s} p_{s}}$.

aligned along the spanwise direction. This alignment indicates that the common structures between $p$ and $w$ fluctuations induce $w$ fluctuations with opposite signs along the $z$ direction. Further, the weak correlations $c_{p_{s} w_{s}}= \pm 0.03$ depicted in Fig. 9(b) indicate that the correlations take different inclinations with respect to the wall [positive and negative correlations are highlighted by black and blue arrows in Fig. 9(b), respectively]. The common structure between $p$ and $w$ fluctuations that could be inferred from the cross-correlation function is the hairpin-type eddy. The legs and the neck of the hairpin eddy are consistent with the different inclinations of the positive and negative correlations. The hairpin eddy configuration was not suggested by Sillero et al. [13] and Jiménez [27]. They recommended the structure associated with $w$ fluctuations to be a quasistreamwise roller that scales in outer units. Note that quasistreamwise rollers were obtained from the correlations of the total $w$ and $v$ fluctuations. Upon computing the correlation length of the pressure fluctuations, Jiménez [27] also stated that these rollers are the dominant structures for the pressure fluctuations. Here, we are concerned with the small scale of motions for $p$ and $w$ fluctuations that show a common $\log$ profile, and these correlations suggest a configuration of the hairpin kind. Additionally, the autocorrelation functions $c_{p_{s} p_{s}}$ and $c_{w_{s} w_{s}}$ presented in Fig. 8 are correlated over the same distances. 


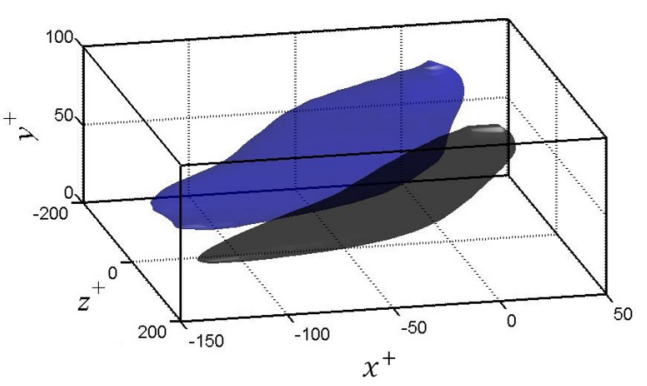

(a)

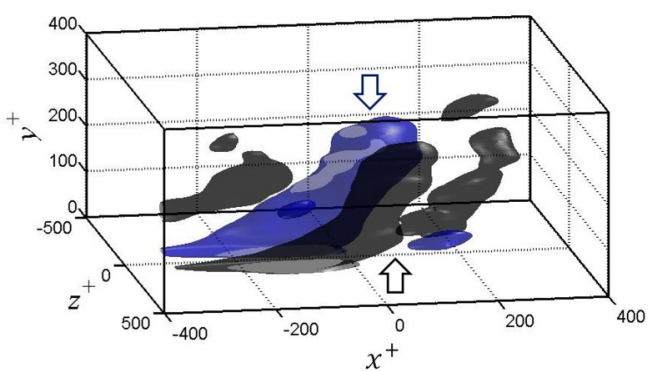

(b)

FIG. 9. Three-dimensional view of the cross-correlation function $c_{p_{s} w_{s}}$ of the small-scale pressurespanwise velocity fluctuations at the reference point $(\tilde{y} / h \approx 0.1)$ for $\operatorname{Re}_{\tau}=1000$. In panel (a), $c_{p_{s} w_{s}}= \pm 0.08$ and in panel (b) $c_{p_{s} w_{s}}= \pm 0.03$. Positive and negative correlations are represented by the black and the blue colors, respectively.

\section{COHERENT STRUCTURES AND CONDITIONAL SAMPLING}

We try to detect the structures that inhabit the channel and are responsible for generating these logarithmic behaviors of $p$ and $w$. The small scales of motion could be represented by the vortex structures across the channel. For vortex identification, we adopt the method of the second invariant of the velocity gradient tensor, the so-called $Q$ criterion developed by Hunt et al. [39] (or $Q^{+}$after inner scaling with wall units). The velocity gradient tensor is written as $D_{i j}=\partial u_{i}^{t} / \partial x_{j}$. As this is a second-order tensor, it can be decomposed into symmetric and skew-symmetric parts $D_{i j}=S_{i j}+\Omega_{i j}$, where $S_{i j}=1 / 2\left(\partial u_{i}^{t} / \partial x_{j}+\partial u_{j}^{t} / \partial x_{i}\right)$ is the rate-of-strain tensor, and $\Omega_{i j}=1 / 2\left(\partial u_{i}^{t} / \partial x_{j}-\partial u_{j}^{t} / \partial x_{i}\right)$ is the rotation tensor. The second invariant tensor $Q$ of the velocity gradient tensor is expressed as

$$
Q=1 / 2\left[\|\Omega\|^{2}-\|S\|^{2}\right] .
$$

The $Q$ criterion defines a vortex as a connected fluid region with a positive second invariant of $D_{i j}$, i.e., $Q>0$. By definition of $Q$, the vortices occur where the rotation magnitude exceeds the rate-of-strain magnitude. Poisson's equation for the total pressure $p^{t}$ is identified as

$$
\nabla^{2} p^{t} / \rho=-\left(\partial u_{i}^{t} / \partial x_{j}\right)\left(\partial u_{j}^{t} / \partial x_{i}\right)
$$

where $\rho$ is the fluid density. The right-hand side of Eq. (10) is written in terms of $S_{i j}$ and $\Omega_{i j}$ (e.g., Bradshaw and Koh [40]) as

$$
\nabla^{2} p^{t} / \rho=-\left[\|\Omega\|^{2}-\|S\|^{2}\right] .
$$

From Eqs. (9) and (11), it is apparent that the $Q$ criterion is directly related to the source term of Poisson's pressure equation.

\section{A. Conditional averaging procedure}

We define the coherent vortex structures by the conditional sampling technique. Following the experimental study of Ghaemi and Scarano [41] and a previous study by some of the authors of the present paper [29], we employ the positive high-amplitude pressure peaks to obtain the conditional eddy structure. In line with the discussion of the previous section where small scales are shown to have significant contributions to the logarithmic profiles, instead of using the positive high-amplitude total pressure at certain $y$ as the detection criterion, the small scale of the pressure $p_{s}$ is utilized.

The first step in the analysis is to decompose the total instantaneous pressure into $p_{s}$ and $p_{l}$. It is noted that the small scales of the pressure have a zero mean, $\left\langle p_{s}\right\rangle=0$. In the next step, a definite 
location from the wall $y_{p}$ is selected ( $y_{p}$ will be referred to as "the detection position"). In the next step, we select multiple wall-normal locations up to $y_{w e}^{+}$. In the third step, when the small-scale pressure fluctuation exceeds its local rms value, $p_{s \mathrm{sms}}$, by more than a certain amount $k_{\text {th }}$, the local extrema (maxima and minima) of the small-scale pressure are detected, and the turbulence quantity $M_{\text {tur }}$ is averaged over part of the DNS computational domain $\left(L_{x}, L_{y}, L_{z}\right)$. We refer to this region as "the calculation domain of the averaging procedure" or simply "the conditioned domain." The reference point is the small-scale pressure located at $\left(x_{\mathrm{ex}}, y_{p}, z_{\mathrm{ex}}\right)$. Thus, the conditionally averaged quantity is sampled for positive fluctuations of the small-scale pressure as

$$
\left\langle M_{\mathrm{tur}}\right\rangle_{p s} \text { such that } p_{s}\left(x_{\mathrm{ex}}, y_{p}, z_{\mathrm{ex}}\right) \geqslant k_{\mathrm{th}} p_{\text {srms }},
$$

where the brackets $\langle\ldots\rangle_{p s}$ refer to conditional averaging by the positive small-scale pressures. As in most previous studies, we fix the threshold value at $k_{\mathrm{th}}=3$. Note that the conditional average by the extrema of the small-scale pressure differs from nonconditional averaging over time and wallparallel plane (in this paper, the nonconditional averages are indicated by the brackets, $\langle\ldots\rangle$, and the conditional averages by $p_{s}$ are denoted by $\langle\ldots\rangle_{p s}$ ). Also, the total pressure and spanwise velocity fluctuations as well as the vortex structures identified by the $Q$ criterion represent the turbulence quantity $M_{\text {tur }}$ that is averaged by the condition of the small-scale pressure fluctuations.

\section{B. Conditional structure of small-scale pressure $p_{s}$}

Figure 10 shows the 3D organization of the vortex structure identified by the $Q$ criterion (transparent black) and normalized in wall units around the high-amplitude positive small-scale pressure fluctuation at $y_{p}^{+}=43$ for $\operatorname{Re}_{\tau}=1000$. The figure provides different views of the structure. The panels are viewed in the inner-scaled coordinate frame. The structure shown in the figure is obtained with the threshold level $\left\langle Q_{\mathrm{th}}^{+}\right\rangle_{p s}=0.0018$. The averaged eddy structure consists of two parts: a downstream part resembling a hairpin eddy, and an upstream part [encircled by the red dashed circle in Fig. 10(c)]. The upstream part is not entirely clear to us, although it seems like a head of another vortex in front of the main vortex. This refers to the existence of a vortex packet within the channel flow. A similar structure was reported by Dennis and Nickels [42] in their turbulent boundary layer flow from experiment.

The downstream hairpin part is approximately symmetric about $z^{+}=0$ and exhibits two streamwise legs, a neck, and a spanwise head. This structure appears to be located close to the wall (within $y^{+} \approx 60$ ), with streamwise and spanwise coherences $\mathcal{O}(150)$ in wall units. The inclination to the wall increases from approximately $8^{\circ}$ for the legs to around $43^{\circ}$ for the neck. These slopes are clarified by the inclinations of the cyan and magenta lines in Fig. 10(b) to the horizontal $x$ axis. The equal streamwise and spanwise extensions of our conditioned hairpin eddy are consistent with the 1D spectral analysis presented previously in Sec. IV. Similar spatial features of the averaged eddy were obtained by Tang et al. [43] and Adrian and Liu [44], who indicated that the mean width of the hairpin eddy is about 100 to 200 in viscous units. The detection scheme they utilized was the maximum ejection event at $y^{+} \approx 49$. With the same detection scheme, Ghaemi and Scarano [41] investigated approximately the same spatial features presented here.

The conditioned hairpin vortex provides the connection between the pressure and spanwise velocity fluctuations at some distances from the wall. Figure 10 shows the isosurfaces of conditioned positive pressure highlighted by the red color $\left(\langle p\rangle_{p s} / p_{\text {rms }}=2.5\right)$ and the conditioned negative pressure highlighted by the blue color $\left(\langle p\rangle_{p s} / p_{\text {rms }}=-0.05\right)$. The value of the negative pressure is lower than that of the positive pressure as the high-amplitude positive small-scale pressure is utilized as the detection criterion. As discussed by Ghaemi and Scarano [41], the positive pressure is located between the two legs of the hairpin eddy. The two legs, the neck, and the spanwise head of the hairpin eddy provide the lift-up mechanism for the low-speed fluid in the near-wall region which results in an ejection event. The ejection motion induced by the hairpin eddy meets a sweep motion from the outer layer that results in a stagnation effect, and hence a positive pressure peak. On the other hand, the negative pressure is associated with the core of the hairpin eddy represented by the 


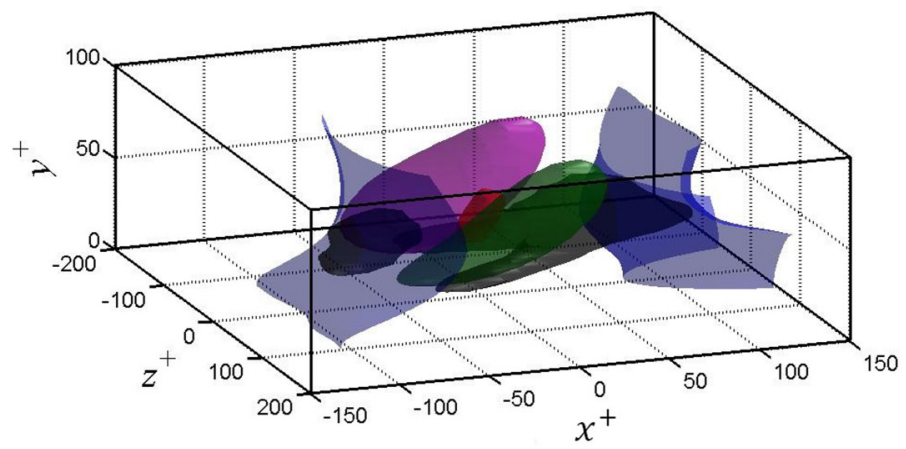

(a)

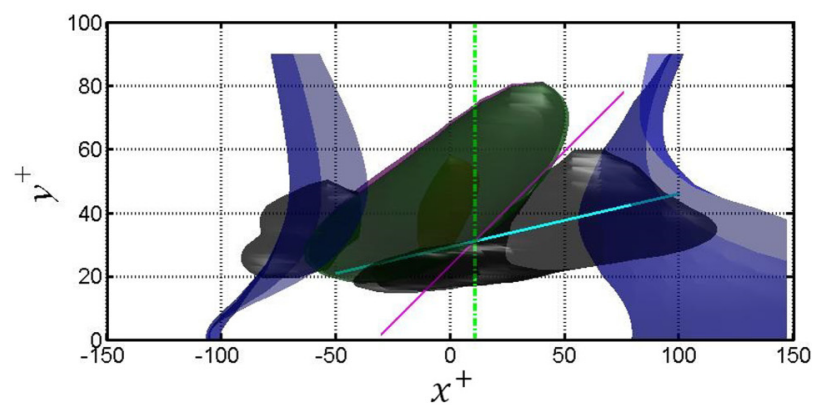

(b)

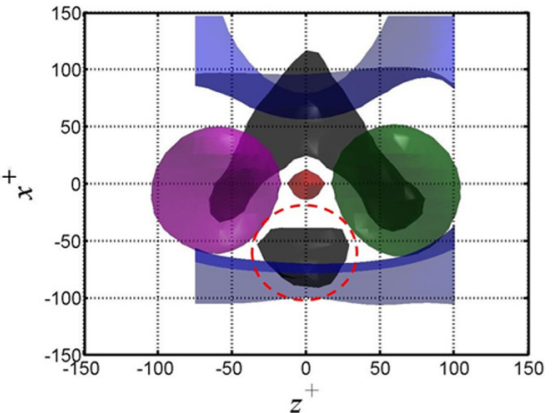

(c)

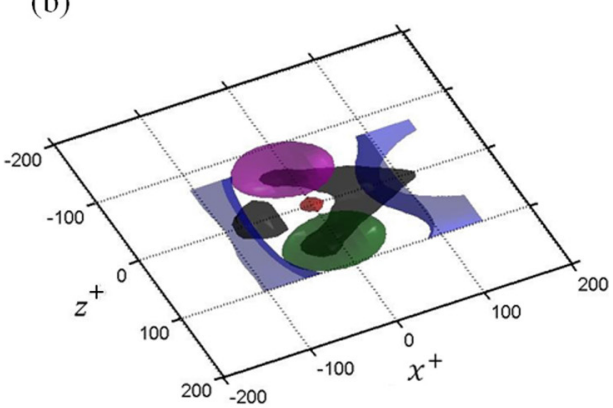

(d)

FIG. 10. Isosurfaces of the vortex organization $\left\langle Q_{\mathrm{th}}^{+}\right\rangle_{p s}=0.0018$ (transparent black), the average unfiltered pressure $\langle p\rangle_{p s} / p_{\text {rms }}=2.5$ (transparent red), $\langle p\rangle_{p s} / p_{\text {rms }}=-0.05$ (transparent blue), and the average unfiltered spanwise velocity $\left\langle w^{+}\right\rangle_{p s}=1$ (transparent green), $\left\langle w^{+}\right\rangle_{p s}=-1$ (transparent magenta) at $\operatorname{Re}_{\tau}=1000$. (a) Three-dimensional view, (b) front view, (c) horizontal view, and (d) oblique view. In panel (b), the cyan and magenta lines indicate the inclinations of the legs and the neck of the eddy, respectively.

head and neck parts. Two negative isosurfaces are presented in the figure, and they are visualized at the value of $\langle p\rangle_{p s} / p_{\text {rms }}=-0.05$. The upstream isosurface of the negative pressure overlaps the upstream vortex structure [encircled by the red dashed circle in Fig. 10(c)]. This reinforces our suggestion that this upstream vortex structure represents a head of another hairpin eddy located much closer to the wall. It is noted that comparable conditioned values of positive and negative pressure associated with the hairpin eddy are obtained if we change the detection scheme of the hairpin eddy. For instance, instead of using the high-amplitude positive pressure, the maximum ejection event criterion can be utilized as reported by Ghaemi and Scarano [41]. Additionally, the 
(a)

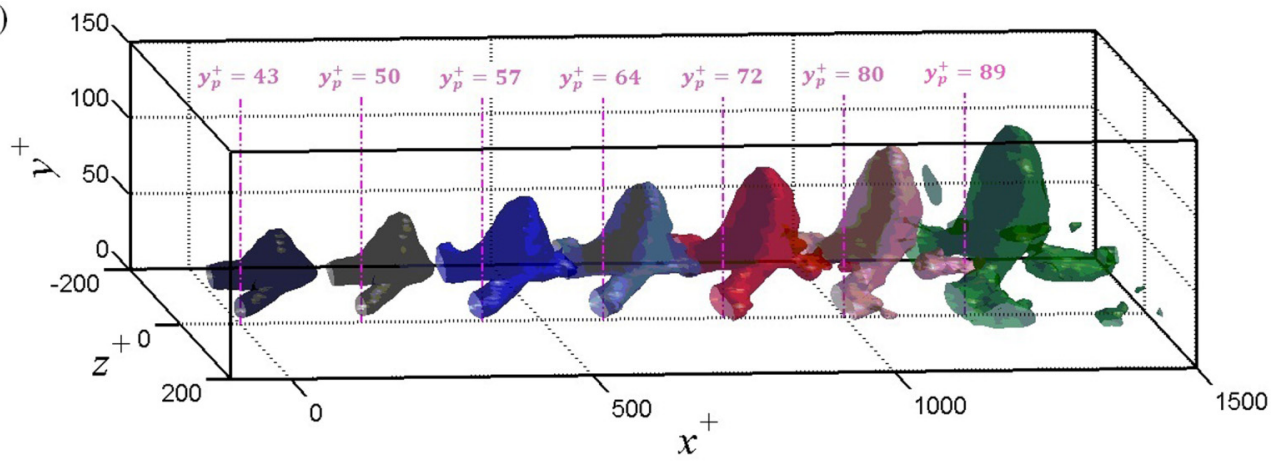

(b)

$+$
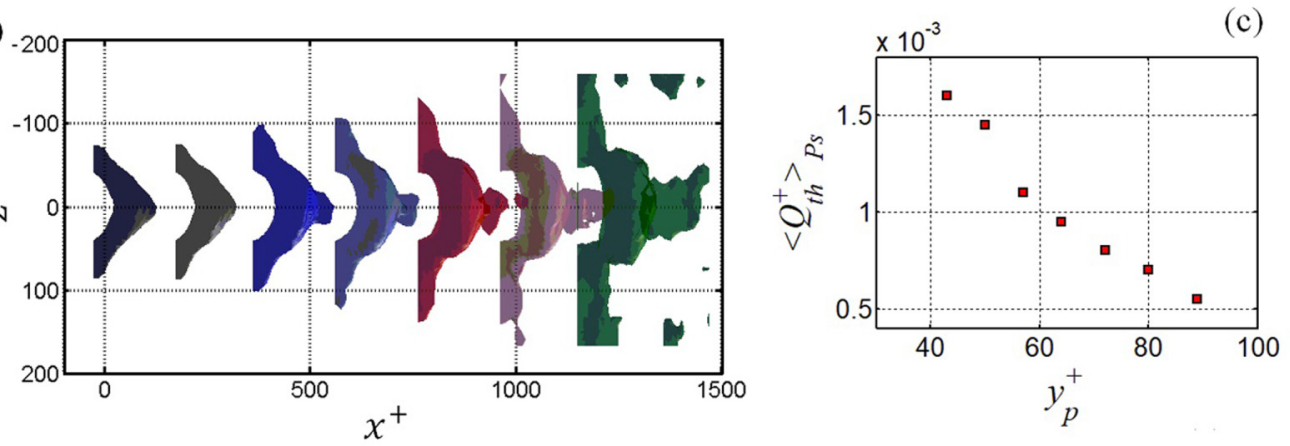

FIG. 11. Isosurfaces of the vortex organization conditioned by the positive $p_{s}$ at different $y_{p}$ 's in (a) threedimensional view, and (b) horizontal view at $\operatorname{Re}_{\tau}=1000$. Coordinates are normalized in wall units. In panel (a), the vertical dash-dotted magenta lines represent $y_{p}$ for each conditioned eddy. (c) The threshold level $\left\langle Q_{\mathrm{th}}^{+}\right\rangle_{p s}$ vs $y_{p}^{+}$.

overlap between the negative pressure and the head and neck parts will be more distinguishable than the results reported here.

Figure 10 also contains the isosurfaces of conditioned positive and negative spanwise velocity which are represented by the values $\left\langle w^{+}\right\rangle_{p s}=1$ (highlighted by the green color) and $\left\langle w^{+}\right\rangle_{p s}=-1$ (indicated by the magenta color). Positive and negative $\left\langle w^{+}\right\rangle_{p s}$ are positioned along the legs and the neck part of the hairpin eddy. Consequently, they are symmetric with respect to the positive pressure point. Apparently, positive and negative $\left\langle w^{+}\right\rangle_{p s}$ result from the counter-rotating motions of the two legs of the hairpin eddy that eject the low-speed fluid. The relation between the pressure fluctuations and the hairpin eddy structures reported here also agrees with the linear model of Luhar et al. [37] where they showed that the impacts of the hairpins are the positive and negative pressure peaks at the wall. Here, we extend this association of the hairpins to the static pressure field and the spanwise velocity fluctuations.

As such, packets of hairpin eddies are associated with, or responsible for, the similar behavior found here between the pressure and spanwise velocity fluctuations. Every packet contains several hairpin eddies similar to that shown in Fig. 10. They could be detected by conditioning the smallscale pressure fluctuations at different wall-normal locations $y_{p}$.

\section{Self-similar eddies: Conditional $p_{s}$ structures at multiple $y_{p}$ locations}

Figure 11 shows the isosurfaces of the conditioned vortex structures by the small-scale pressure at different values of $y_{p}$. The following procedure was employed to create Fig. 11. First, we isolate the hairpin eddy results from the conditional sampling at each $y_{p}$. We did not account for the upstream vortex structure [encircled by the dashed red circle in Fig. 10(c)] as we are mainly concerned with 
the hairpin eddy structures. So, this upstream part is not displayed in Fig. 11 to reduce clutter. Second, the size of the vortex structure increases with increasing $y_{p}$. Hence, the calculation domain for the averaging process had to be increased as well to correctly capture the size of the conditioned eddy. However, we have not plotted the full extent of the fields. We have just displayed the part that contains the hairpin eddy. Third, to make the results sensible, we plot the conditioned vortex structures obtained from the different $y_{p}$ 's on the same axis. They are discriminated from each other by different colors. This procedure is similar to the one employed by Elsinga [45], Dennis and Nickels [42], and Tang et al. [43]. In addition, the vertical dash-dotted magenta lines shown in the figure clarify the detection position $y_{p}$ of each individual eddy. Finally, the results are shown in an inner-scaled frame of the coordinates.

The threshold level $\left\langle Q_{\mathrm{th}}^{+}\right\rangle_{p s}$ required to visualize the averaged structure varies with $y_{p}$. Utilizing a uniform threshold level for all $y_{p}$ 's leads to elimination of many of the details of the hairpin eddy as we go further away from the wall. The scheme we utilized is to change $\left\langle Q_{\mathrm{th}}^{+}\right\rangle_{p s}$, such that results from the small-scale positive pressure at each $y_{p}$ lead to a hairpin eddy. $\left\langle Q_{\mathrm{th}}^{+}\right\rangle_{p s}$ is plotted versus $y_{p}^{+}$ in Fig. 11(c). Del Álamo et al. [46] pointed out this issue when discussing the vortex clusters in the turbulent logarithmic region. They reported that utilizing a uniform threshold level is not appropriate to visualize the clusters due to the inhomogeneity of the flow in the wall-normal direction. Further, Nagaosa and Handler [47] recommended normalizing the threshold value by its local rms value, $Q_{\mathrm{rms}}$, when visualizing the vortex in the viscous sublayer. We tried to visualize the structures by normalizing $\left\langle Q_{\mathrm{th}}^{+}\right\rangle_{p s}$ with its local rms, $\left\langle Q_{\mathrm{th}}^{+}\right\rangle_{p s} / Q_{\mathrm{rms}}$, but we did not obtain a significant change in the results as we are far from the viscous sublayer.

Figure 11 shows clearly the different hairpin eddies detected at the different positions up to $y_{p}^{+}=89$. From Fig. 11(a), it is observed that the neck and head part reaches wall-normal locations higher than $y_{p}$. For example, the neck and head part of the hairpin eddy at $y_{p}^{+}=89$ approaches $y^{+} \approx 150$. This means that even though the hairpin eddy is located or detected at a definite $y_{p}$ its effect may extend to several wall-normal locations. Hence, the log trend of the variances of $p$ and $w$ may result from structures detected outside the ranges $\left[y_{p s}, y_{p e}\right]$ and $\left[y_{w s}, y_{w e}\right]$ shown in Figs. 1(b) and 2(b), respectively.

The overall size of the eddies increases with increasing the distance from the wall. However, the shape does not change. On the other hand, the structures could be visualized in a frame of coordinates that are normalized by the detection wall-normal position $y_{p}$. Data in Fig. 11 are now replotted with the axes normalized with $y_{p}$ and are shown in Fig. 12. The interesting feature here is that the size of the structure becomes almost invariant. This means that the streamwise, spanwise, and wall-normal extensions of the hairpin eddy scale with $y_{p}$. Hence, the indicated eddies represent "geometrically self-similar eddies." So, from the statistical point of view, the log trends of the variances of $p$ and $w$ are related to these self-similar eddies.

\section{Self-similar eddies extending to overlap and outer layers}

The structures shown in Figs. 11 and 12 are limited to $y_{p}^{+}=89$ (with the $y_{p}^{+}=89$ eddy extending up to $y^{+} \approx 150$ or $y / h \approx 0.15$ ). Beyond this location, it is very difficult to obtain a clear hairpin eddy as the two legs vanish and we are just left with the neck and head part of the hairpin eddy. This may be consistent with the study of Robinson [48], as he indicated that the "arch" type or the spanwise head of the hairpin eddy exists in the outer layer. Accordingly, these geometrically shaped self-similar eddies could be characterized by the neck and head part of the hairpin eddy even at $y_{p}^{+}$ less than $y_{p}^{+}=89$. For the detected structures close to the wall up to $y_{p}^{+}=89$, the neck and head part of the hairpin eddy is isolated by following the scheme which is shown in Fig. 10(b). At first, the hairpin is viewed from the front view. Second, the inclinations to the wall of the legs and neck and head part of the hairpin eddy are evaluated by the cyan and magenta lines, respectively. Third, the point at which the inclination changes represents the "cutting" point, and the vertical dash-dotted line indicates the isolating section. 

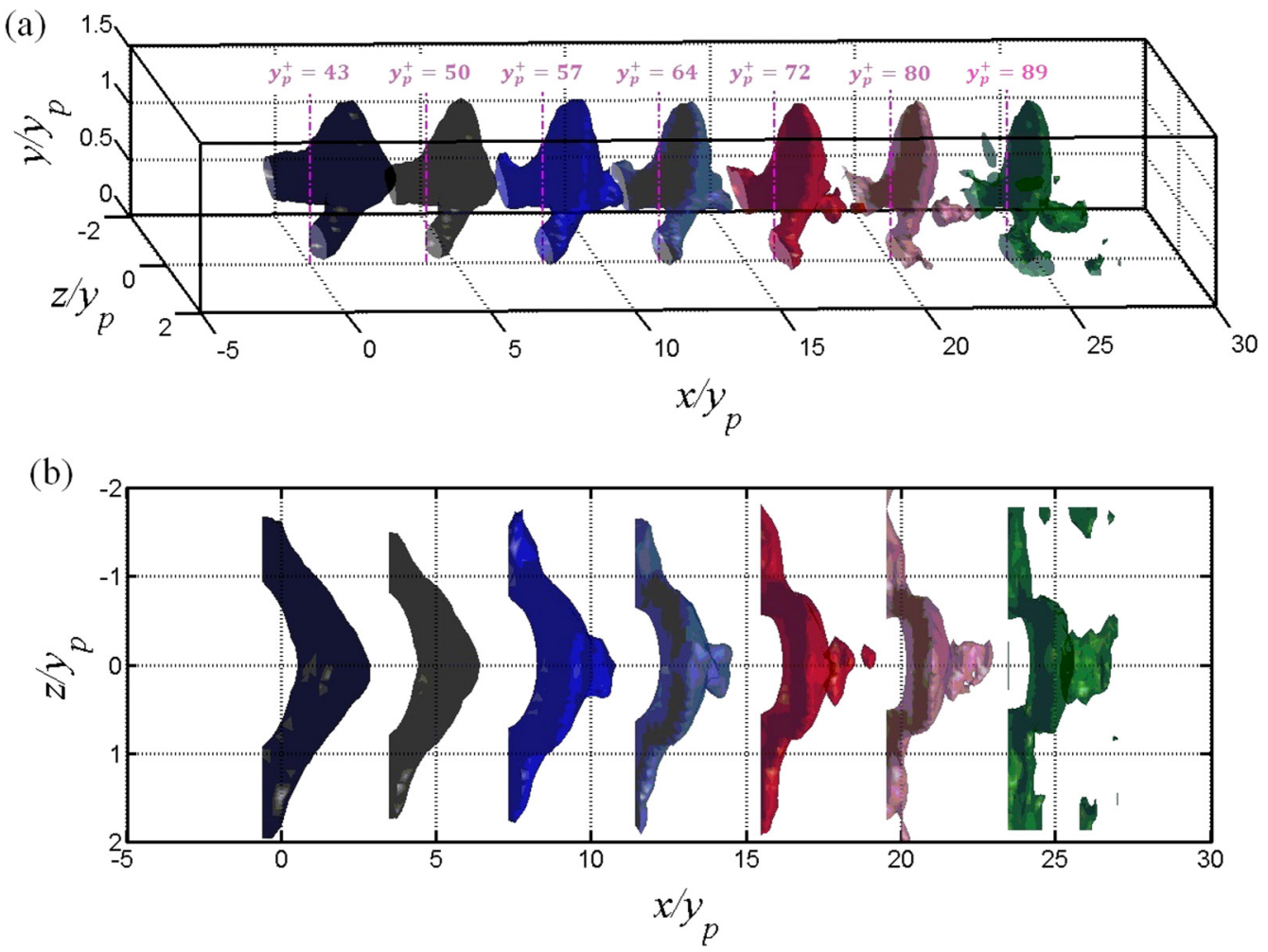

FIG. 12. Same as in Figs. 11(a) and 11(b); however, now the axes are scaled with $y_{p}$ rather than viscous units.

The different structures of the neck and head part are shown in Fig. 13. They are displayed on the same axis with different colors, and the magenta vertical dash-dotted lines characterize $y_{p}^{+}$for each conditioned eddy ( $y_{p}^{+}$is selected from $y_{p}^{+}=43$ to 221). The length and the width of the neck and head part are indicated by $\Delta x_{\text {eddy }}^{+}$and $\Delta z_{\text {eddy }}^{+}$, as shown in Figs. 13(b) and 13(c), respectively. They are defined by the bounding box of the eddy structure, following the same procedure of Sillero et al. [13] and Del Álamo et al. [46]. $\Delta x_{\text {eddy }}^{+}$and $\Delta z_{\text {eddy }}^{+}$increase with the distance from the wall. When plotted versus $y_{p}^{+}, \Delta x_{\text {eddy }}^{+}$and $\Delta z_{\text {eddy }}^{+}$follow linear relations with $y_{p}^{+}$as shown in Fig. 13(d) (square symbols represent $\Delta z_{\text {eddy }}^{+}$versus $y_{p}^{+}$, while circles represent $\Delta x_{\text {eddy }}^{+}$versus $y_{p}^{+}$). As a result, when $\Delta x_{\text {eddy }}^{+}$is plotted versus $\Delta z_{\text {eddy }}^{+}$in Fig. 13(e), we obtain a linear relation between $\Delta x_{\text {eddy }}^{+}$and $\Delta z_{\text {eddy }}^{+}$. This linear relation signifies the feature of the geometric self-similar shape of the structures located at definite wall-normal locations through the channel. In addition, the overall size of the eddies in streamwise, wall-normal and spanwise directions scales with $y_{p}$. This is shown in Fig. 13(a) where the coordinates are normalized with $y_{p}$. Each individual eddy is scaled as $\mathcal{O}\left(\Delta x_{\text {eddy }}=y_{p}, y_{p}\right.$, $\left.\Delta z_{\text {eddy }}=1.2 y_{p}\right)$. The threshold level for the $Q$ criterion, $\left\langle Q_{\mathrm{th}}^{+}\right\rangle_{p s}$, is plotted versus $y_{p}^{+}$in Fig. 13(f).

The Reynolds number effect is presented in Fig. 14. For $\operatorname{Re}_{\tau}=1000,2000$, and $4000,\left\langle Q_{\mathrm{th}}^{+}\right\rangle_{p s}$ is plotted versus $y_{p}^{+}$in panel (a). Increasing Reynolds number leads to a decrease in the threshold level required to detect the structures. This may be reasonable from the fact that smaller scales of motion appear with the higher Reynolds number. Hence, to detect the same structures as those detected at lower Reynolds number in the conditional sampling, the threshold values have to be decreased. However, it is observed that we need to do that in the near-wall region. Beyond $y_{p}^{+} \approx 200$ the threshold level approaches asymptotically a constant value. 

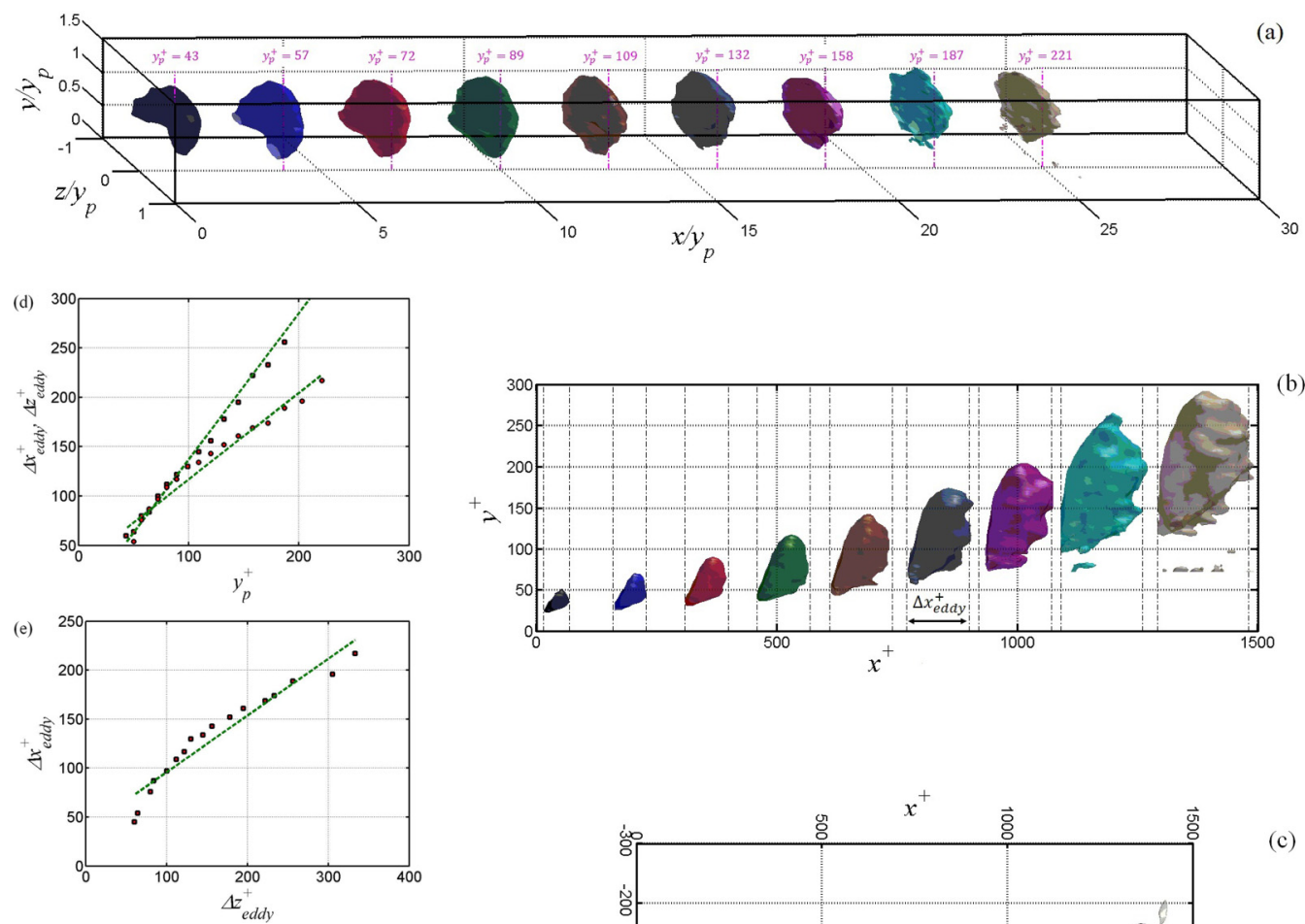

(b)
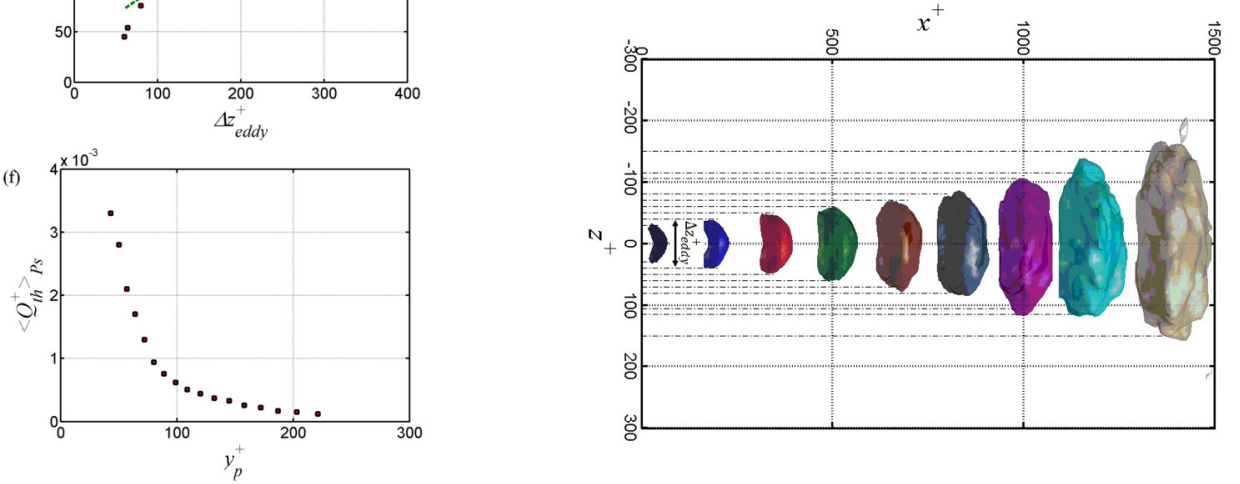

FIG. 13. Isosurfaces of the vortex organization conditioned by the positive $p_{s}$ at different $y_{p}$ 's in (a) threedimensional view, (b) front view, and (c) horizontal view at $\operatorname{Re}_{\tau}=1000$. Coordinates are normalized by $y_{p}$ in panel (a), and in wall units in panels (b) and (c). In panel (a), the dash-dotted magenta vertical lines represent $y_{p}$ for each conditioned eddy. In panel (d), the length $\Delta x_{\text {eddy }}^{+}$(circles) and the width $\Delta z_{\text {eddy }}^{+}$(squares) of the eddy are plotted vs $y_{p}^{+}$, and they are plotted vs each other in panel (e). (f) The threshold level $\left\langle Q_{\mathrm{th}}^{+}\right\rangle_{p s}$ vs $y_{p}^{+}$.

In Fig. 14(b), the scaling of $\Delta x_{\text {eddy }}^{+}$(circles) and $\Delta z_{\text {eddy }}^{+}$(squares) with the distance from the wall $y_{p}^{+}$is displayed for the different Reynolds numbers. The linear fittings between $\Delta x_{\text {eddy }}^{+}$and $\Delta z_{\text {eddy }}^{+}$ and $y_{p}^{+}$are plotted with the dashed blue lines. Clearly, the self-similar structures are confirmed for this range of Reynolds numbers since $\Delta x_{\text {eddy }}^{+}$and $\Delta z_{\text {eddy }}^{+}$fall on straight lines with slopes of approximately 0.78 and 1.16 , respectively. Also, it is observed that $\Delta z_{\text {eddy }}^{+}$is larger than $\Delta x_{\text {eddy }}^{+}$. This is shown in Fig. 14(c) where $\Delta z_{\text {eddy }}^{+}$and $\Delta x_{\text {eddy }}^{+}$are plotted versus each other.

\section{CONCLUSION}

The scope of this paper is to explore the log behavior of the pressure variance and higher-order moments as well as the related coherent structures. Acknowledging that the spanwise velocity $w$ shows its logarithmic intensity profile at the same Reynolds numbers, we extend our analysis 

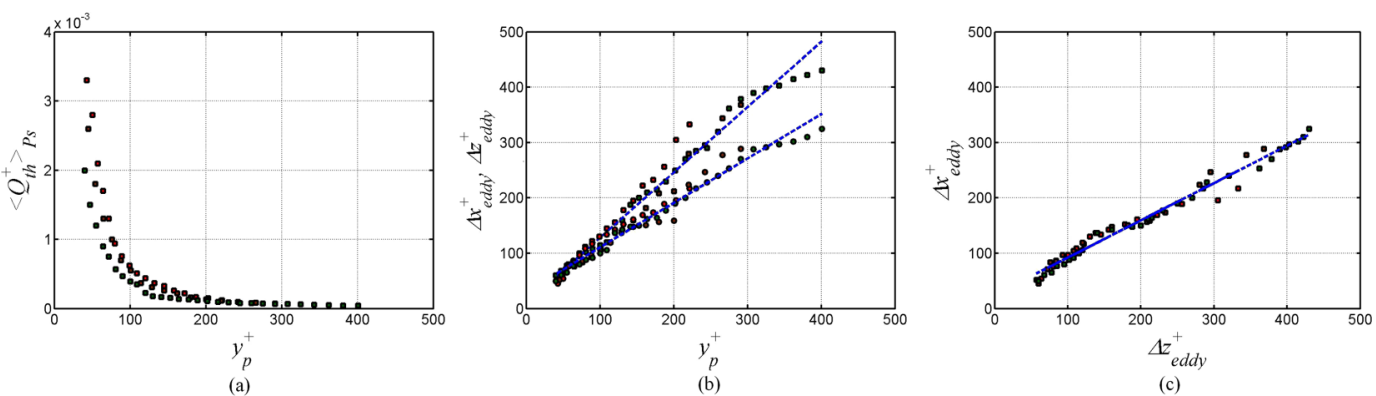

(b)

(c)

FIG. 14. (a) The threshold level $\left\langle Q_{\mathrm{th}}^{+}\right\rangle_{p s}$ vs $y_{p}^{+}$. (b) The length of the eddy $\Delta x_{\text {eddy }}^{+}$vs $y_{p}^{+}$(circles), and the width of the eddy $\Delta z_{\text {eddy }}^{+}$vs $y_{p}^{+}$(squares). The lower blue dashed line is the linear fitting between $\Delta x_{\text {eddy }}^{+}$and $y_{p}^{+}$, and the upper one is the linear fitting between $\Delta z_{\text {eddy }}^{+}$and $y_{p}^{+}$with slopes of $\approx 0.78$ and 1.16, respectively. (c) $\Delta x_{\text {eddy }}^{+}$vs $\Delta z_{\text {eddy }}^{+}$with the linear fitting represented by the blue dashed line with a slope of $\approx 0.66$. In each panel, the results are plotted for $\operatorname{Re}_{\tau}=1000$ (red), $\operatorname{Re}_{\tau}=2000$ (brown), and $\operatorname{Re}_{\tau}=4000$ (green).

to $w$ and discuss the similarity between the pressure and spanwise velocity fluctuations. For this investigation, we utilize DNS datasets of fully developed turbulent channel flow from $\operatorname{Re}_{\tau}=180$ to 4000.

The pressure variance starts its logarithmic profiles at a wall-normal location of approximately 150 in wall units, while the log profile of the spanwise velocity variance starts closer to the wall at around 90 in wall units, independent of Reynolds number. The upper bounds of both profiles show linear relations with $\operatorname{Re}_{\tau}$ with the same slope of around 0.2. The log regions of $\left\langle p^{+^{2}}\right\rangle$ and $\left\langle w^{+^{2}}\right\rangle$ overlap each other over significant portions of the wall distance, and the minimum Reynolds number estimated for both $\log$ regions to be identified is $\operatorname{Re}_{\tau} \approx 500$. Additionally, we have studied the higher-order moments of $p$ and $w$, where the $2 n$-order moments raised to the power of $1 / n$, with $n=$ $1, \ldots, 4$, follow the logarithmic behaviors within the same regions where $\left\langle p^{+^{2}}\right\rangle$ and $\left\langle w^{+^{2}}\right\rangle$ confirm the log profiles. In addition, the higher-order moments of $p$ and $w$ follow super-Gaussian behaviors which reinforce the similarity between $p$ and $w$. The implication of the logarithmic scaling of the higher-order moments of $p$ and $w$ fluctuations is their association with a hierarchical organization of self-similar eddies.

The coherent structures related to the pressure intensity log profile, and hence the spanwise velocity, are also investigated. The instantaneous fields of $p$ and $w$ are decomposed into small and large scales of motion with sharp spectral filters $\lambda_{x c}=\lambda_{z c}=0.7 \mathrm{~h}$ which are associated with the ridge of the spectra at the upper bound of the $\left\langle w^{+^{2}}\right\rangle \log$ profile. The small scales of motion showed that they are the main contributors to the log trends of $p$ and $w$. We used the conditional sampling technique to explore the structures associated with these small scales of motion. The conditional sampling around the positive high-amplitude small-scale pressure fluctuations at different wallnormal locations results in hairpin eddy structures. Positive pressure fluctuations are located between the legs of the hairpin eddy, while the negative pressure fluctuations are consistent with the head part of the hairpin eddy. Positive and negative spanwise velocity fluctuations are strongly positioned consistently with the legs and neck of the hairpin eddy. The eddy structure shows a strong link between $p$ and $w$ fluctuations, both of which occur at relatively smaller scales. Furthermore, these small-scale structures are found to be geometrically self-similar. Their length and width increase linearly with the distance from the wall. As such, the size of the eddy does not change when it is scaled with the distance from the wall.

\section{ACKNOWLEDGMENTS}

This research used computational resources of the SX-ACE provided by Tohoku University through the HPCI System Research project (Project No. hp170195), and the Earth Simulator Pro- 
posed Research Projects of Japan Agency for Marine-Earth Science and Technology (JAMSTEC). Financial support from Japan Society for the Promotion of Science (B) Grant No. 15H03917 is gratefully acknowledged. J.P. also acknowledges financial support from the Australian Research Council.

\section{APPENDIX A: WALL-NORMAL EXTENT OF THE LOG BEHAVIORS IN $\left\langle p^{+^{2}}\right\rangle$ AND $\left\langle w^{+^{2}}\right\rangle$}

Here, we compare the log regions of $\left\langle p^{+^{2}}\right\rangle$ and $\left\langle w^{+^{2}}\right\rangle$ with each other. For this, we replot the insets of Figs. 1(b) and 2(b) in Fig. 15. As seen in the figure, $\left\langle w^{+^{2}}\right\rangle$ starts its logarithmic profile closer to the wall than $\left\langle p^{+^{2}}\right\rangle$, whereas $\left\langle p^{+^{2}}\right\rangle$ maintains a log trend to higher wall-normal locations than $\left\langle w^{+^{2}}\right\rangle$. Interestingly, both upper bounds $y_{p e}^{+}$and $y_{w e}^{+}$are parallel to each other as they have approximately the same slope. Generally, the log region of $\left\langle w^{+^{2}}\right\rangle$ is much narrower than that of $\left\langle p^{+^{2}}\right\rangle$. However, they overlap each other over significant portions of their lengths, and this overlap increases with Reynolds number.

From Fig. 15, the minimum Reynolds number $\operatorname{Re}_{\tau \min p}$ at which $\left\langle p^{+^{2}}\right\rangle$ possesses its logarithmic profile could be estimated. As indicated previously, the upper bound of the $\left\langle p^{+^{2}}\right\rangle$ log profile is related to the Reynolds number as $y_{p e}^{+}=0.204 \mathrm{Re}_{\tau}+395.6$. If the condition for the existence of the $\left\langle p^{+^{2}}\right\rangle \log$ region is restricted to $y_{p e}^{+} \geqslant y_{p s}^{+} \approx 150$, this yields an unphysical negative $\operatorname{Re}_{\tau \min p}$. Hence, $\operatorname{Re}_{\tau \min p}$ must be at least higher than the intercept 395.6. The value of $\operatorname{Re}_{\tau \min p} \approx 500$ guarantees the existence of the $\left\langle p^{+^{2}}\right\rangle \log$ region with an upper bound of $y_{p e}^{+} \approx 497.6$. Although there are no studies to which we can directly compare this minimum $\operatorname{Re}_{\tau}$, there exists the work of Farabee and Casarella [8] that has looked at the dependence of the wall pressure variance $\left\langle p_{w}^{+^{2}}\right\rangle$ on $\operatorname{Re}_{\tau}$. In turbulent boundary layers, they found the minimum $\operatorname{Re}_{\tau} \approx 333$, and the relation for wall pressure variance is $\left\langle p_{w}^{+^{2}}\right\rangle=6.5+1.86 \ln \left(\operatorname{Re}_{\tau} / 333\right)$.

The minimum Reynolds number $\operatorname{Re}_{\tau} \min w$ for the $\left\langle w^{+^{2}}\right\rangle \log$ profile can be obtained directly from the condition $y_{w e}^{+} \geqslant y_{w s}^{+} \approx 90$, yielding $\operatorname{Re}_{\tau \min w} \approx 340$, which is a little bit lower than $\operatorname{Re}_{\tau \min p}$. This difference between $\operatorname{Re}_{\tau \min p}$ and $\operatorname{Re}_{\tau \min w}$ can be attributed to the lower bound of the $\left\langle w^{+^{2}}\right\rangle \log$ region which is below that of $\left\langle p^{+^{2}}\right\rangle$. Hence, a very narrow plateau region can be noticed in Fig. 2(b) for $\beta_{w w}$ at $\operatorname{Re}_{\tau}=400$. However, this plateau region is obviously distinguishable for higher Reynolds

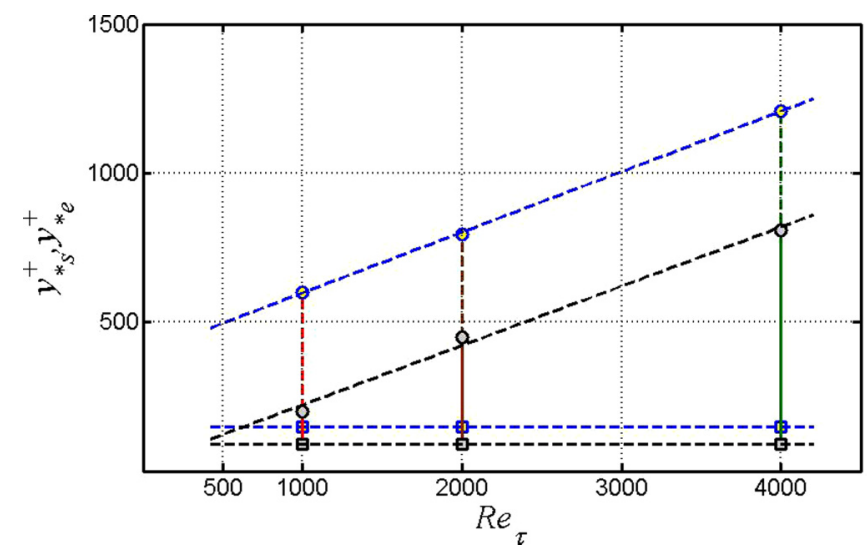

FIG. 15. Replot of the insets in Figs. 1(b) and 2(b). The subscript $*$ in the label of the $y$ axis, $y_{*_{s}}^{+}, y_{*_{e}}^{+}$, stands for both $p$ and $w$. Yellow squares and circles represent $y_{p s}^{+}$and $y_{p e}^{+}$, respectively at $\operatorname{Re}_{\tau}=1000,2000$, and 4000. Gray squares and circles indicate $y_{w s}^{+}$and $y_{w e}^{+}$, respectively at the same Reynolds numbers. The horizontal dashed blue line represents $y_{p s}^{+}=150$, and the black one indicates $y_{w s}^{+}=90$. The inclined dashed blue line represents $y_{p e}^{+}=0.204 \mathrm{Re}_{\tau}+395.6$, and the black one indicates $y_{w e}^{+}=0.2 \mathrm{Re}_{\tau}+22.3$. 


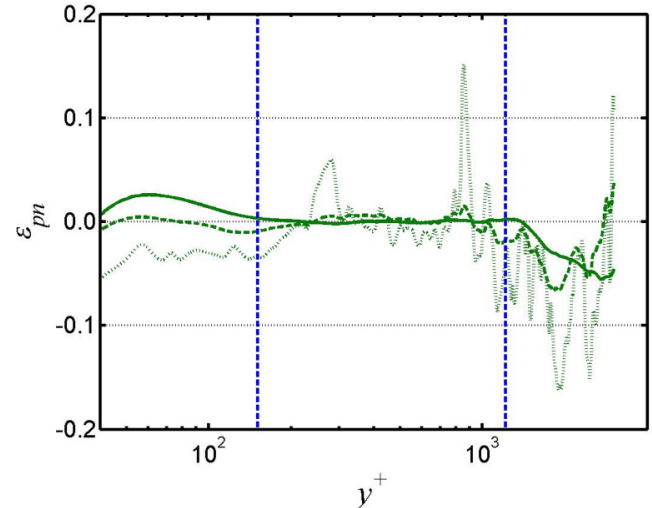

(a)

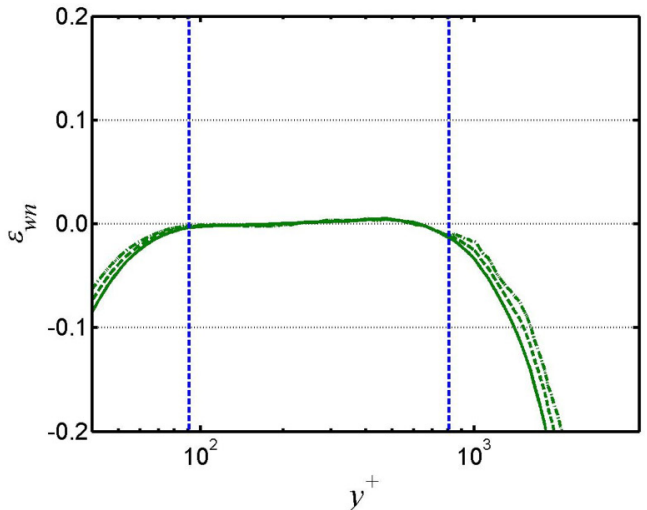

(b)

FIG. 16. Relative error for the linear fittings of $2 n$-order moments in inner variable $y^{+}$

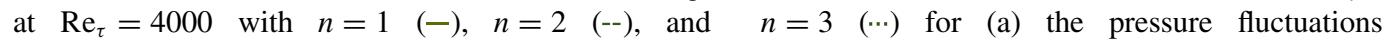
$\varepsilon_{p n}=\left\{\left\langle p^{+2 n}\right\rangle^{1 / n}-\left[-A_{p_{n}} \ln (y / h)+B_{p_{n}}\right]\right\} /\left\langle p^{+2 n}\right\rangle^{1 / n}$, and (b) spanwise velocity fluctuations $\varepsilon_{w n}=$ $\left\{\left\langle w^{+2 n}\right\rangle^{1 / n}-\left[-A_{w_{n}} \ln (y / h)+B_{w_{n}}\right]\right\} /\left\langle w^{+2 n}\right\rangle^{1 / n}$ up to $n=4$ (-.). The blue vertical dashed lines are corresponding to $y_{p s}^{+}$and $y_{p e}^{+}$in panel (a), and $y_{w s}^{+}$and $y_{w e}^{+}$in panel (b).

numbers. Thus, it could be inferred that the minimum Reynolds numbers for $\left\langle p^{+^{2}}\right\rangle$ and $\left\langle w^{+^{2}}\right\rangle \log$ profiles are approximately the same $\mathcal{O}(500)$.

\section{APPENDIX B: STATISTICAL CONVERGENCE CHECKS FOR EQS. (5) AND (6)}

In order to show more clearly the high quality of these logarithmic fittings, the relative errors of the fittings for the pressure and spanwise velocity fluctuations are defined as

$$
\begin{gathered}
\varepsilon_{p n}=\left\{\left\langle p^{+2 n}\right\rangle^{1 / n}-\left[-A_{p_{n}} \ln (y / h)+B_{p_{n}}\right]\right\} /\left\langle p^{+2 n}\right\rangle^{1 / n}, \\
\varepsilon_{w n}=\left\{\left\langle w^{+2 n}\right\rangle^{1 / n}-\left[-A_{w_{n}} \ln (y / h)+B_{w_{n}}\right]\right\} /\left\langle w^{+2 n}\right\rangle^{1 / n} .
\end{gathered}
$$

They are displayed in Figs. 16(a) and 16(b) for $n=1,2$, and 3 for the pressure, and up to $n=4$ for the spanwise velocity at $\operatorname{Re}_{\tau}=4000$. The figure indicates that the relative error of pressure fittings is low as it does not exceed $\pm 5 \%$ within the region bounded by $y_{p s}^{+}$and $y_{p e}^{+}$except for $n=3$. On the other hand, the relative error $\varepsilon_{w n}$ of the fittings for the higher-order moments of $w$ does not exceed $\pm 2 \%$ inside the interval $\left[y_{w s}^{+}, y_{w e}^{+}\right]$even for $n=4$, as observed in Fig. 16(b). Again, this refers to the fact that the higher-order moments of $w$ have better statistical convergence than those of $p$.

The statistical convergence is significant when investigating the higher-order moments. Meneveau and Marusic [28] and Yang et al. [49] tested for convergence by evaluating the premultiplied PDF. Following their analysis, the premultiplied PDFs of the pressure $p^{+2 n} F\left(p^{+}\right)$and spanwise velocity fluctuations $w^{+2 n} F\left(w^{+}\right)$are related to the even-order moments of $p$ and $w$ through the relations

$$
\begin{aligned}
\left\langle p^{+^{2 n}}\right\rangle & =\int_{p^{+}} p^{+2 n} F\left(p^{+}\right) d p^{+}, \\
\left\langle w^{+^{2 n}}\right\rangle & =\int_{w^{+}} w^{+^{2 n}} F\left(w^{+}\right) d w^{+} .
\end{aligned}
$$




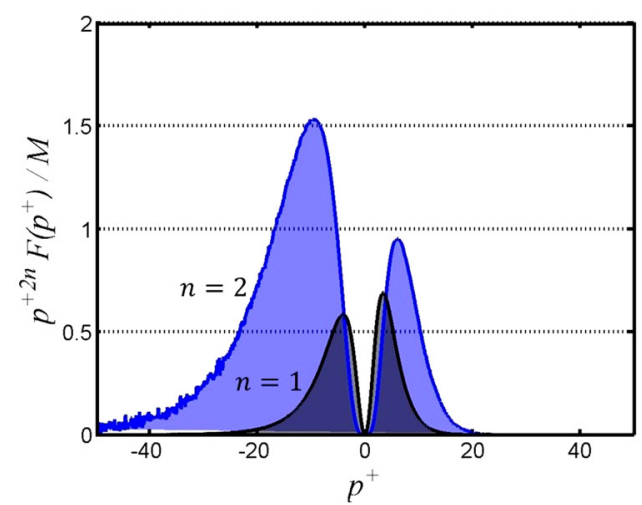

(a)

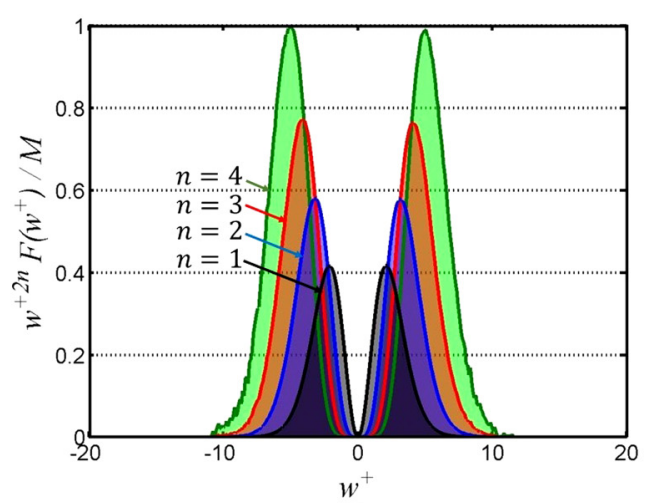

(b)

FIG. 17. Premultiplied PDF of (a) pressure fluctuations $p^{+2 n} F\left(p^{+}\right)$and (b) spanwise velocity fluctuations $w^{+2 n} F\left(w^{+}\right)$for $\operatorname{Re}_{\tau}=4000$ at $y^{+}=150$ with $n=1(-), n=2(-), n=3(-)$, and $n=4(-)$. Premultiplied PDFs are divided by an arbitrary factor $M$ for clarity.

Here, $F\left(p^{+}\right)$and $F\left(w^{+}\right)$are the PDFs of $p$ and $w$, respectively. Hence, the area under the curve of the premultiplied PDF approximates the value of the even-order moment. For instance, we examine $p^{+2 n} F\left(p^{+}\right)$at $y^{+}=150$ for $\operatorname{Re}_{\tau}=4000$, where the premultiplied PDFs are shown in Fig. 17(a) for the pressure for $n=1$ and 2. Evaluating the value of the fourth-order moment of the pressure $\left\langle p^{+^{4}}\right\rangle$ from Eq. (B3) and comparing it with the value of $\left\langle p^{+^{4}}\right\rangle$ obtained from the ensemble averaging, $\langle\ldots\rangle$, of $p^{+^{4}}$ from our DNS leads to an error of about $20 \%$. Accordingly, the error in estimating the square root of the fourth-order moment $\left\langle p^{+^{4}}\right\rangle^{1 / 2}$ is about $4.5 \%$. Following the same procedure for the spanwise velocity, the errors in estimating the values of $\left\langle w^{+^{4}}\right\rangle$ and $\left\langle w^{+^{4}}\right\rangle^{1 / 2}$ are around 3 and $1.8 \%$, respectively.

[1] A. A. Townsend, The Structure of Turbulent Shear Flow (Cambridge University, Cambridge, England, 1976).

[2] A. E. Perry, S. Henbest, and M. S. Chong, A theoretical and experimental study of wall turbulence, J. Fluid Mech. 165, 163 (1986).

[3] N. Hutchins and I. Marusic, Evidence of very long meandering features in the logarithmic region of turbulent boundary layers, J. Fluid Mech. 579, 1 (2007).

[4] I. Marusic and J. P. Monty, Attached eddy model of wall turbulence, Annu. Rev. Fluid Mech. 51, 49 (2019).

[5] J. Jiménez and S. Hoyas, Turbulent fluctuations above the buffer layer of wall-bounded flows, J. Fluid Mech. 611, 215 (2008).

[6] Y. Tsuji, I. Marusic, and A. V. Johansson, Amplitude modulation of pressure in turbulent boundary layer, Int. J. Heat Fluid Fl. 61, 2 (2016).

[7] W. Willmarth, Pressure fluctuations beneath turbulent boundary layers, Annu. Rev. Fluid Mech. 7, 13 (1975).

[8] T. M. Farabee and M. J. Casarella, Spectral features of wall pressure fluctuations beneath turbulent boundary layers, Phys. Fluids A 3, 2410 (1991).

[9] M. Bull, Wall-pressure fluctuations beneath turbulent boundary layers: some reflections on forty years of research, J. Sound Vib. 190, 299 (1996). 
[10] Y. Tsuji, J. H. M. Fransson, P. H. Alfredsson, and A. V. Johansson, Pressure statistics and their scaling in high-Reynolds-number turbulent boundary layers, J. Fluid Mech. 585, 1 (2007).

[11] Z. Hu, C. L. Morfey, and Neil D. Sandham, Wall pressure and shear stress spectra from direct simulations of channel flow, AIAA J. 44, 1541 (2006).

[12] J. A. Sillero, J. Jiménez, and R. D. Moser, One-point statistics for turbulent wall-bounded flows at Reynolds numbers up to $\delta^{+} \approx 2000$, Phys. Fluids 25, 105102 (2013).

[13] J. A. Sillero, J. Jiménez, and R. D. Moser, Two-point statistics for turbulent boundary layers and channels at Reynolds numbers up to $\delta^{+} \approx 2000$, Phys. Fluids 26, 105109 (2014).

[14] M. Hultmark, M. Vallikivi, S. C. C. Bailey, and A. J. Smits, Turbulent Pipe Flow at Extreme Reynolds Numbers, Phys. Rev. Lett. 108, 094501 (2012).

[15] I. Marusic, J. P. Monty, M. Hultmark, and A. J. Smits, On the logarithmic region in wall turbulence, J. Fluid Mech. 716, R3 (2013).

[16] N. Hutchins and I. Marusic, Large-scale influences in near-wall turbulence, Phil. Trans. R. Soc. A 365, 647 (2007).

[17] Y. Yamamoto and Y. Tsuji, Numerical evidence of logarithmic regions in channel flow at $\operatorname{Re}_{\tau}=8000$, Phys. Rev. Fluids 3, 012602 (2018).

[18] H. Fernholz and P. Finleyt, The incompressible zero-pressure-gradient turbulent boundary layer: An assessment of the data, Prog. Aerosp. Sci. 32, 245 (1996).

[19] J. M. österlund, Ph.D. thesis, Royal Institute of Technology, 1999.

[20] R. Baidya, J. Philip, N. Hutchins, J. P. Monty, and I. Marusic, Measurements of streamwise and spanwise fluctuating velocity components in a high Reynolds number turbulent boundary layer, in Proceedings of the 18th Australasian Fluid Mechanics Conference, Launceston, Australia, edited by P. A. Brandner and P. W. Pearce (The Australasian Fluid Mechanics Society, University of Tasmania, 2012), p. 168.

[21] J. Philip, R. Baidya, N. Hutchins, J. P. Monty, and I. Marusic, Spatial averaging of streamwise and spanwise velocity measurements in wall-bounded turbulence using $\vee$ - and $\chi$-probes, Meas. Sci. Technol. 24, 115302 (2013).

[22] C. M. de Silva, Kevin, R. Baidya, N. Hutchins, and I. Marusic, Large coherence of spanwise velocity in turbulent boundary layers, J. Fluid Mech. 847, 161 (2018).

[23] J. Jiménez and R. D. Moser, What are we learning from simulating wall turbulence?, Phil. Trans. R. Soc. A 365, 715 (2007).

[24] J. Jiménez, Near-wall turbulence, Phys. Fluids 25, 101302 (2013).

[25] M. Lee and R. D. Moser, Direct numerical simulation of turbulent channel flow up to $\operatorname{Re}_{\tau} \approx 5200$, J. Fluid Mech. 774, 395 (2015).

[26] R. L. Panton, M. Lee, and R. D. Moser, Correlation of pressure fluctuations in turbulent wall layers, Phys. Rev. Fluids 2, 094604 (2017).

[27] J. Jiménez, Coherent structures in wall-bounded turbulence, J. Fluid Mech. 842, 1 (2018).

[28] C. Meneveau and I. Marusic, Generalized logarithmic law for high-order moments in turbulent boundary layers, J. Fluid Mech. 719, R1 (2013).

[29] A. A. Mehrez, Y. Yamamoto, and Y. Tsuji, Reynolds number dependence of turbulent structures associated with high-amplitude wall pressure peaks in channel flow, Fluid Dyn. Res. 51, 011407 (2019).

[30] Y. Yamamoto and T. Kunugi, Direct numerical simulation of a high-Froude-number turbulent openchannel flow, Phys. Fluids 23, 125108 (2011).

[31] Y. Yamamoto and T. Kunugi, MHD effects on turbulent dissipation process in channel flows with an imposed wall-normal magnetic field, Fusion Eng. Des. 109-111, 1137 (2016).

[32] Z. Xia, G. Brethouwer, and S. Chen, High-order moments of streamwise fluctuations in a turbulent channel flow with spanwise rotation, Phys. Rev. Fluids 3, 022601 (2018). 
[33] X. I. A. Yang, I. Marusic, and C. Meneveau, Hierarchical random additive process and logarithmic scaling of generalized high order, two-point correlations in turbulent boundary layer flow, Phys. Rev. Fluids $\mathbf{1}$, 024402 (2016).

[34] J. Kim, On the structure of pressure fluctuations in simulated turbulent channel flow, J. Fluid Mech. 205, 421 (1989).

[35] R. Baidya, J. Philip, N. Hutchins, J. P. Monty, and I. Marusic, Distance-from-the-wall scaling of turbulent motions in wall-bounded flows, Phys. Fluids 29, 020712 (2017).

[36] H.-P. Wang, S.-Z. Wang, and G.-W. He, The spanwise spectra in wall-bounded turbulence, Acta Mech. Sinica 34, 452 (2018).

[37] M. Luhar, A. Sharma, and B. McKeon, On the structure and origin of pressure fluctuations in wall turbulence: Predictions based on the resolvent analysis, J. Fluid Mech. 751, 38 (2014).

[38] H. Abe, R. A. Antonia, and S. Toh, Large-scale structures in a turbulent channel flow with a minimal streamwise flow unit, J. Fluid Mechs. 850, 733 (2018).

[39] J. C. R. Hunt, A. A. Wray, and P. Moin, Eddies, streams, and convergence zones in turbulent flows, in Proceedings of 1988 Summer Program, Center for Turbulence Research (Stanford University, Stanford, 1988), p. S 193.

[40] P. Bradshaw and Y. Koh, A note on Poisson's equation for pressure in a turbulent flow, Phys. Fluids 24, 777 (1981).

[41] S. Ghaemi and F. Scarano, Turbulent structure of high-amplitude pressure peaks within the turbulent boundary layer, J. Fluid Mech. 735, 381 (2013).

[42] D. J. C. Dennis and T. B. Nickels, Experimental measurement of large-scale three-dimensional structures in a turbulent boundary layer. Part 1. Vortex packets, J. Fluid Mech. 673, 180 (2011).

[43] Z.-Q. Tang, N. Jiang, A. Schröder, and R. Geisler, Tomographic PIV investigation of coherent structures in a turbulent boundary layer flow, Acta Mech. Sinica 28, 572 (2012).

[44] R. J. Adrian and Z. C. Liu, Observation of vortex packets in direct numerical simulation of fully turbulent channel flow, J. Visual-Japan 5, 9 (2002).

[45] G. E. Elsinga, Ph.D. thesis, Delft University of Technology, 2008.

[46] J. C. Del Álamo, J. Jiménez, P. Zandonade, and R. D. Moser, Self-similar vortex clusters in the turbulent logarithmic region, J. Fluid Mech. 561, 329 (2006).

[47] R. Nagaosa and R. A. Handler, Statistical analysis of coherent vortices near a free surface in a fully developed turbulence, Phys. Fluids 15, 375 (2003).

[48] S. K. Robinson, Coherent motions in the turbulent boundary layer, Annu. Rev. Fluid Mech. 23, 601 (1991).

[49] X. I. A. Yang, I. Marusic, and C. Meneveau, Moment generating functions and scaling laws in the inertial layer of turbulent wall-bounded flows, J. Fluid Mech. 791, R2-1 (2016). 